\title{
Beam Loss Studies for Rare Isotope Driver Linacs Final Report
}

FY07 DOE Grant Number LAB 06-29

\section{March 26, 2008}

T. P. Wangler, S. S. Kurennoy, J. H. Billen, K. R. Crandall, J. Qiang, R. D. Ryne, B. Mustapha, P. Ostroumov, Q. Zhao, and R. C. York

\subsection{Introduction}

The Fortran 90 RIAPMTQ/IMPACT code package is a pair of linked beam-dynamics simulation codes that have been developed for end-to-end computer simulations of multiple-charge-state heavy-ion linacs for future exotic-beam facilities. These codes have multiple charge-state capability, and include space-charge forces. The simulations can extend from the low-energy beam-transport line after an ECR ion source to the end of the linac. The work has been performed by a collaboration including LANL, LBNL, ANL, and MSU. The code RIAPMTQ simulates the linac front-end beam dynamics including the LEBT, RFQ, and MEBT. The code IMPACT simulates the beam dynamics of the main superconducting linac. The codes have been benchmarked for rms beam properties against previously existing codes at ANL and MSU. The codes allow high-statistics runs on parallel supercomputing platforms, particularly at NERSC at LBNL, for studies of beam losses. The codes also run on desktop PC computers for low-statistics work. The code package is described in more detail in a recent publication [1] in the Proceedings of PAC07 (2007 US Particle Accelerator Conference).

In this report we describe the main activities for the FY07 beam-loss studies project using this code package. These activities included the following FY07 work.

1) We completed implementation of the beam-steering-correction capability in IMPACT to provide realistic corrections to beam-centroid displacements caused by misaligned focusing elements. This capability is based on the use of steering magnets and beam-position monitors. This capability is necessary for realistic beam-loss studies. Details of the procedure used in IMPACT are given in Appendix A by Ji Qiang.

2) The capability of introducing random machine errors was implemented in IMPACT. This capability is necessary for the beam-loss studies.

3) We performed simulation studies with random errors including beam-loss calculations for two RIA design examples using the RIAPMTQ/IMPACT code

\footnotetext{
${ }^{1}$ T. P. Wangler, R. W. Garnett, J. Qiang, R. Ryne, K. R. Crandall, J. H. Billen, V. N. Aseev, B. Mustapha, P. Ostroumov, M. Doleans, D. Gorelov, X. Wu, R. C. York, and Q. Zhao, "The RIAPMTQ/IMPACT Beam-Dynamics Simulation Package", Proc. of PAC07, Albuquerque, NW, USA (2007) pp. 3606-3608.
} 
package. One of these was provided by ANL and the other was provided by MSU. Neither of these design examples was the final RIA design, but both represented intermediate steps in the design process and were expected to be representative of good designs with low beam losses. The main objective of our work was to confirm this low beam-loss expectation for both designs.

4) We completed users manuals for both codes, RIAPMTQ and IMPACT.

5) We completed this report on the beam loss studies.

The beam-loss studies with random errors involved repeated simulation runs of the RIAPMTQ and IMPACT codes at the LBNL NERSC high-performance computing facility using random errors for beam elements and cavity fields, and including automatic steering compensation for the beam centroid, compensating for random focusing-element misalignments. We chose to study beam losses for these two RIA design examples, even though there is no plan at present to build the RIA machine, because we already had complete input files for these designs that we used in the previous code-development work. Furthermore, the machine-lattice architecture of the RIA machine is still representative of the machine that is expected to be built, although the final energy of such a machine will be reduced to half that of the previous RIA specification. In other words beam losses would be an even more important concern for any RIA design than for the scaled-down lower-energy machine that is expected to be built. We anticipate that the factor of 2 reduced final energy from $400 \mathrm{MeV} / \mathrm{u}$ for RIA to $200 \mathrm{MeV} / \mathrm{u}$ will relax the beam-loss specification based on activation at the final energy by about a factor of 4 .

After an initial step where we did 10 different runs with different random number sets, to ensure that things were working properly for the NERSC runs, we made 100 runs with random errors and with steering corrections with $200 \mathrm{~K}$ simulation particles per run. Machine error tolerances were estimated from previous accelerator projects and were put into the input file that included the machine errors. For our initial study, the error tolerances (plus or minus values) used were quadrupole and solenoid displacements at each the end of the element $=0.2 \mathrm{~mm}$, quadrupole rotations $=0.3 \mathrm{deg}$, quadrupole and solenoid field amplitudes $=1 \%$, cavity displacement $=0.5 \mathrm{~mm}$, cavity RF field amplitude $=1 \%$, cavity phase $=1 \mathrm{deg}$, and beam-position monitor rms error $=0.2 \mathrm{~mm}$. These are tight error tolerances, but are expected to be feasible. Then, 100 RIAPMTQ runs, beginning just before the multiharmonic buncher, with different random misalignments and field errors within the error-tolerance window, were made at NERSC. The output coordinates were passed on to IMPACT. The IMPACT runs, also with random machine errors, transported the beam particles to the end.

The results for the 100 RIAPMTQ runs for the MSU design example are shown in the plots in Section 2.0, and for the 100 runs for the ANL design example in the plots in Section 3.0. The results for the 100 IMPACT runs for the MSU design example are shown in the plots in Section 4.0, and for the ANL design example in the plots in Section 5.0. A summary and discussion of the beam losses is presented in Section 6.0. Appendix 
A presents the methods implemented in IMPACT for including errors and steering corrections.

\subsection{Calculating the power loss per meter from the simulations}

\subsection{Calculating the initial number of particles per bunch}

First we need to calculate the initial number of real particles per bunch, which we call

R. In RIAPMTQ we read in the electrical current for each of the two charge states $(q=28$ and $\mathrm{q}=29$ ) as $0.125 \mathrm{~mA}$ each. This is the current for each charge state at the bunch frequency $\mathrm{f}_{\mathrm{b}}$, which is $28.75 \mathrm{MHz}$ for ANL and $40.25 \mathrm{MHz}$ for MSU, each being half the RFQ frequency, $57.5 \mathrm{MHz}$ for ANL and $80.5 \mathrm{MHz}$ for MSU. The current I, the number of real particles per bunch $\mathrm{R}$, and the bunch frequency are related by

$$
I=q R f_{b}
$$

For ANL the initial number of real particles per bunch for $\mathrm{q}=28$ is

$$
R=0.000125 /\left(1.602 \times 10^{-19} \times 28 \times 28.75 \times 10^{6}\right)=969.3 K
$$

and $R=935.9 K$ for $\mathrm{q}=29$. The initial number per bunch corresponding to the mean charge state of $\mathrm{q}=28.5$ for ANL is $R=952.6 K$.

For MSU the initial number of real particles per bunch for $\mathrm{q}=28$ is

$$
R=0.000125 /\left(1.602 \times 10^{-19} \times 28 \times 40.25 \times 10^{6}\right)=688.1 K
$$

and $R=664.4 K$ for $\mathrm{q}=29$. The initial number per bunch corresponding to the mean charge state of $\mathrm{q}=28.5$ for $\mathrm{MSU}$ is $R=676.3 \mathrm{~K}$.

Here, we note that in the RFQ, where the frequency is twice the bunch frequency $(57.5$ $\mathrm{MHz}$ for ANL and $80.5 \mathrm{MHz}$ for MSU), the number of particles per bunch has not changed, every bucket is filled, and the current has doubled to $0.25 \mathrm{~mA}$.

In the RFQ the $\mathrm{q}=28$ particles and $\mathrm{q}=29$ particles populate alternate buckets. It will be convenient for purposes of calculating power losses from lost particles to simplify matters by assuming that all buckets in the RFQ contain the same numbers of real particles, whose initial value is that corresponding to the mean charge state $\mathrm{q}=28.5$. From the above results we have $R=952.6 K$ for ANL and $R=676.3 K$ for MSU. We also note that the numbers for $\mathrm{R}$ will change along the linac, mostly from particle losses in the RFQ, and as a result of what happens at the strippers. There will also be particle losses along the linac, which we will assume for now do not decrease R very much. This is discussed in Sections 3 and 4. 


\subsection{Basic formulas for beam power loss}

Next, suppose that at kinetic energy E, we have S simulation particles per bunch, which will correspond to whatever we chose in our simulations, and $\mathrm{R}$ real particles per bunch.

If we loose $\mathrm{N}_{\mathrm{R}}$ real particles in a given element of length, the watts lost in that element is

$$
\begin{aligned}
& P(\text { Watts per element })=N_{R} f_{b}(\mathrm{~Hz}) E(\mathrm{eV}) \times 1.6 \times 10^{-19}(\mathrm{~J} / \mathrm{eV}) \\
& =N_{R} f_{b}(\mathrm{MHz}) E(\mathrm{MeV}) \times 1.6 \times 10^{-7}(\mathrm{~J} / \mathrm{eV})
\end{aligned}
$$

From the simulation, we know the number of lost simulation particles. Call this $\mathrm{N}_{\mathrm{S}}$.

We can assume that $N_{R}=N_{S} R / S$, and we can write

$$
P(\text { Watts per element })=(R / S) N_{S} f_{b}(M H z) E(M e V) \times 1.6 \times 10^{-7}
$$

To get Watts per meter for a given element, we divide by which is length in meters for that element. Then

$$
\frac{P(\text { Watts })}{\ell(m)}=\frac{1.6 \times 10^{-7} \times(R / S) N_{S} f_{b}(M H z) E(M e V)}{\ell(m)}
$$

This is the basic formula for calculating Watts per meter beam loss from the simulation. We are not quite done yet. We know $\mathrm{N}_{\mathrm{S}}$ and $\mathrm{S}$ because these numbers come from the simulation, and we have calculated the initial $R$ value, but how do we get $R$ and $R / S$ at different points along the linac? This requires knowing what happens in the strippers. This is calculated in Sections 2.0 and 3.0 and displayed in Tables 1 and 2.

\subsection{MSU results for number of real particles per bunch $R$ along the linac}

For MSU we have already calculated the initial value of $\mathrm{R}$ in Section 1, corresponding to the mean charge state of $\mathrm{q}=28.5$, which is $\mathrm{R}=676.3 \mathrm{MHz}$ for MSU.

Usually we would expect that $\mathrm{R} / \mathrm{S}$ is constant along the linac. But, in this case with a stripper, it is not necessarily constant because after the stripper, we don't change the number of particles in the simulation, but the real number does change. The RFQ transmission is $83 \%$. Looking at the stripper data, I found that the first stripper at about $12 \mathrm{MeV} / \mathrm{u}$ has about $80 \%$ transmission, and the second stripper has approximately $100 \%$ transmission. So the results for $\mathrm{R} / \mathrm{S}$ along the linac are given in Table 1, where I have assumed the initial number of simulation particles is $200 \mathrm{~K}$ and I have assumed that I can ignore the few lost particles at high energy for calculating the number of simulated 
particles per bunch $\mathrm{S}$. At high energies, the ratio that we need to scale the simulation power loss results to real power loss results is $R / S=2.70$.

Table $1 \quad$ MSU values of number of particles $\mathrm{R}$ and $\mathrm{S}$ per bunch

\begin{tabular}{|c|c|c|c|}
\hline Location & $\mathrm{R}$ & $\mathrm{S}$ & $\mathrm{R} / \mathrm{S}$ \\
\hline Input to RFQ & $676 \mathrm{~K}$ & $200 \mathrm{~K}$ & 3.38 \\
\hline $\begin{array}{l}\text { After RFQ }(0.293 \\
\text { MeV/amu) }\end{array}$ & $561 \mathrm{~K}$ & 166 & 3.38 \\
\hline $\begin{array}{l}\text { After } 1^{\text {st }} \text { stripper (12 } \\
\mathrm{MeV} / \mathrm{u})\end{array}$ & $449 \mathrm{~K}$ & 166 & 2.70 \\
\hline
\end{tabular}

\subsection{ANL results for number of real particles per bunch $R$ along the linac}

For ANL we have already calculated in Section 1.0 the initial value of $\mathrm{R}$ corresponding to the mean charge state of $\mathrm{q}=28.5$ as $952.6 \mathrm{~K}$. The same considerations discussed in Section 3.0 apply here as well. At high energies the ratio that we need to scale the simulation power loss results to real power loss results is $\mathrm{R} / \mathrm{S}=3.81$.

Table 2 ANL values of number of particles $\mathrm{R}$ and $\mathrm{S}$ per bunch

\begin{tabular}{|l|l|l|l|}
\hline Location & $\mathrm{R}$ & $\mathrm{S}$ & $\mathrm{R} / \mathrm{S}$ \\
\hline Input to RFQ & $953 \mathrm{~K}$ & $200 \mathrm{~K}$ & 4.76 \\
\hline $\begin{array}{l}\text { After RFQ }(0.293 \\
\text { MeV/amu) }\end{array}$ & $791 \mathrm{~K}$ & 166 & 4.76 \\
\hline $\begin{array}{l}\text { After } 1^{\text {st }} \\
\text { MeV/u) }\end{array}$ & $633 \mathrm{~K}$ & 166 & 3.81 \\
\hline
\end{tabular}

\subsection{Results of 100 RIAPMTQ runs for the MSU design example}

The plots below compare results for an MSU lattice from 100 runs with random errors and steering with the results from a run without errors or steering, all with $200 \mathrm{~K}$ particles. 


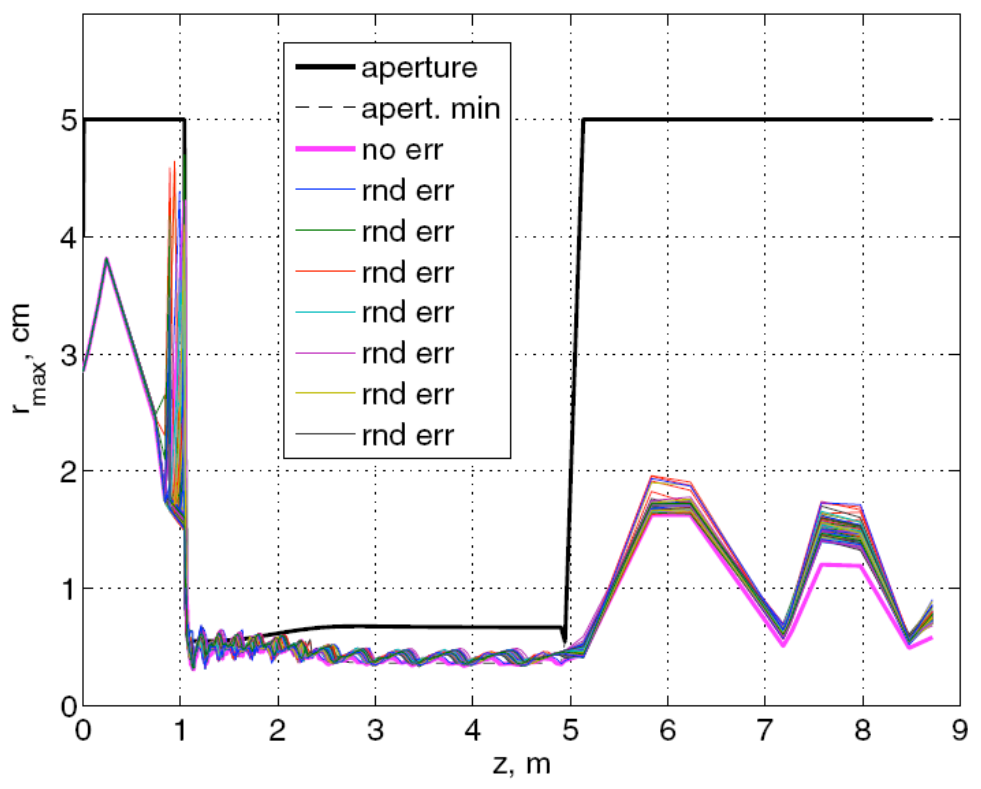

Fig. 3-1: MSU lattice: Beam maximal radius versus distance along the beam line in meters without random errors and steering (thick line, magenta) and with random errors and steering, 100 lines (cycling colors) where each line corresponds to a given run. The aperture is also shown in black. 

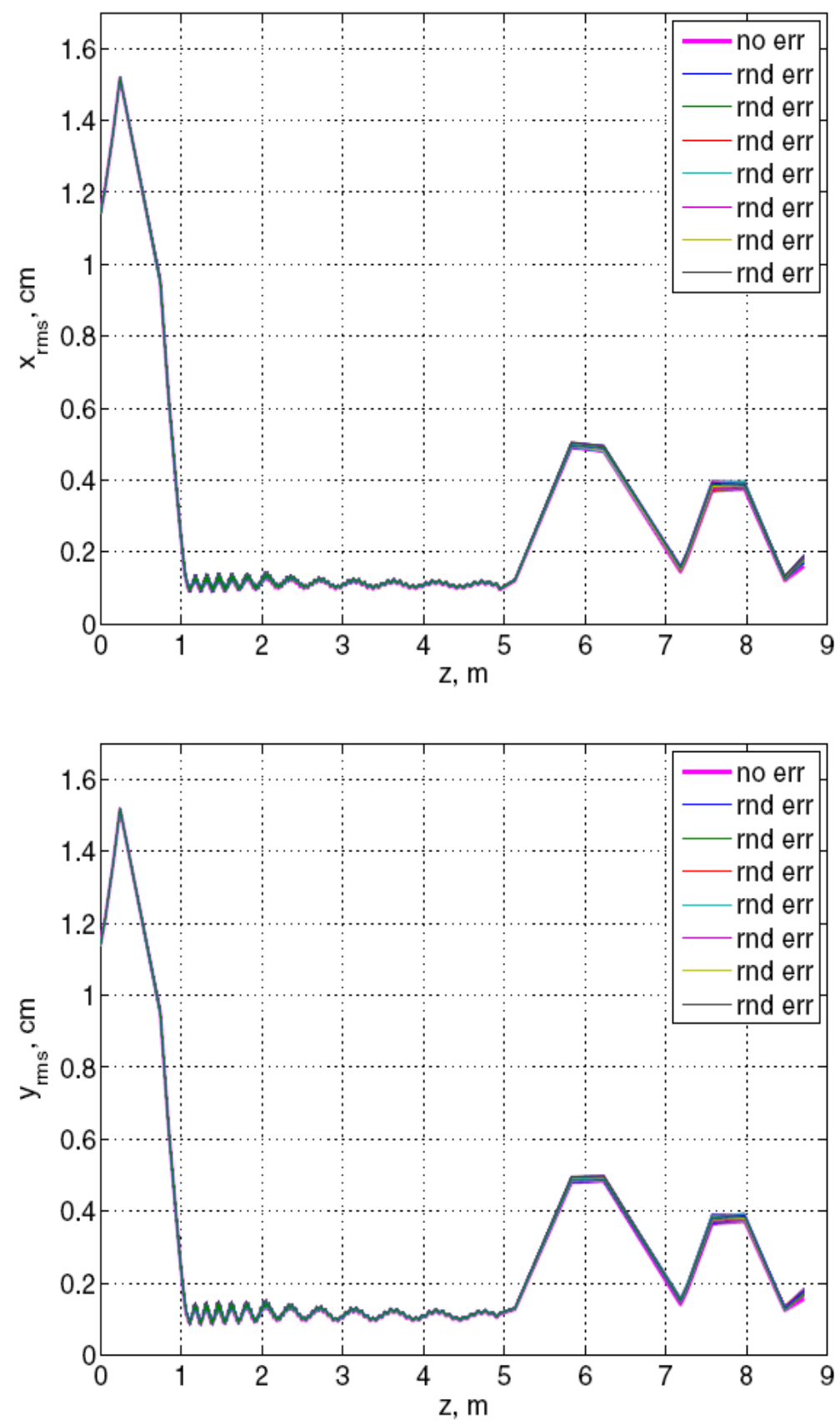

Fig. 3-2: MSU lattice: Beam rms sizes in $\mathrm{x}$ and y versus distance along the beam line in meters without random errors and steering (thick line, magenta) and with random errors and steering, 100 lines (cycling colors) where each line corresponds to a given run. 

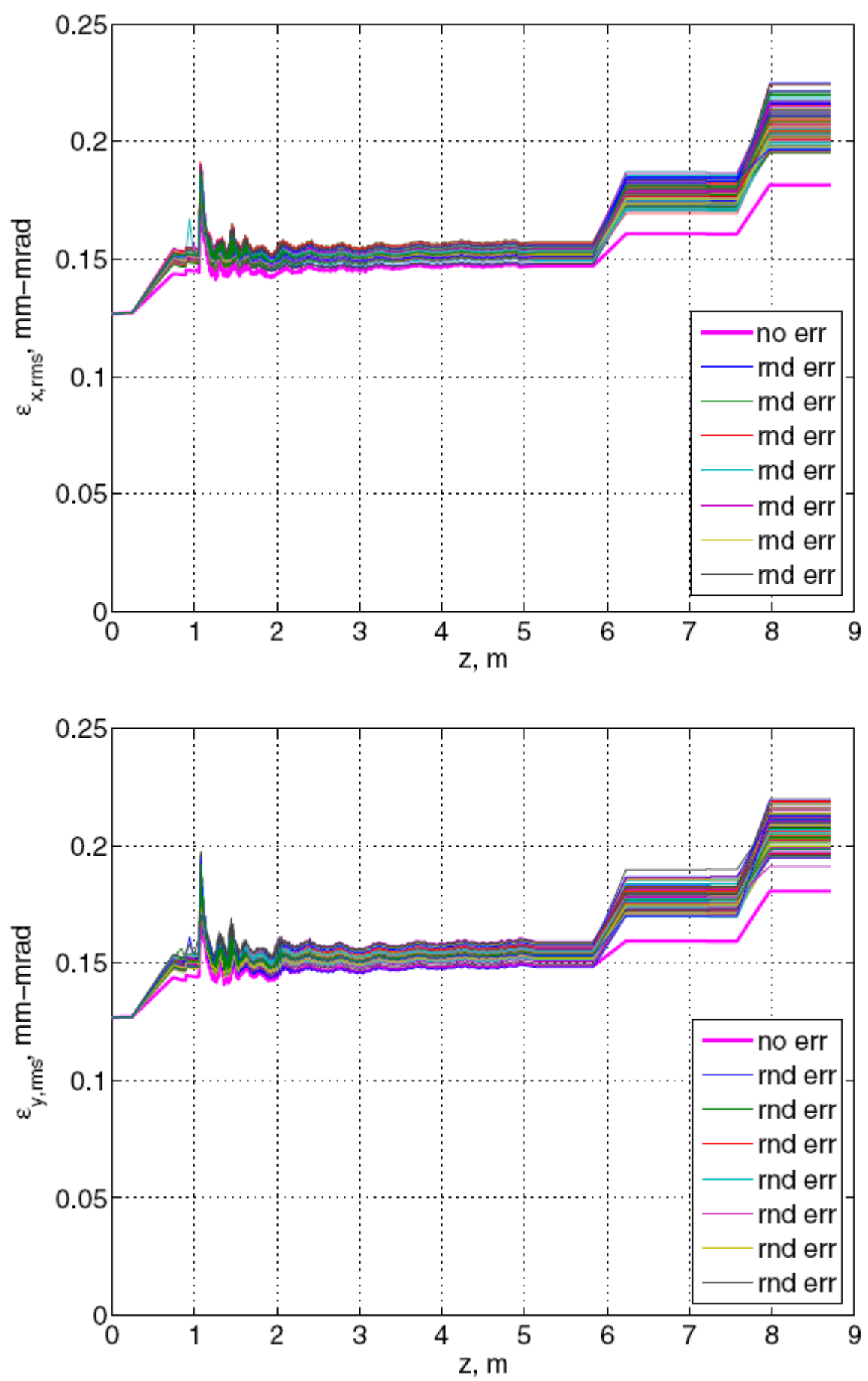

Fig. 3.3 MSU lattice: Beam rms emittances in $\mathrm{x}$ and $\mathrm{y}$ versus distance along the beam line in meters without random errors and steering (thick line, magenta) and with random errors and steering, 100 lines (cycling colors) where each line corresponds to a given run. 

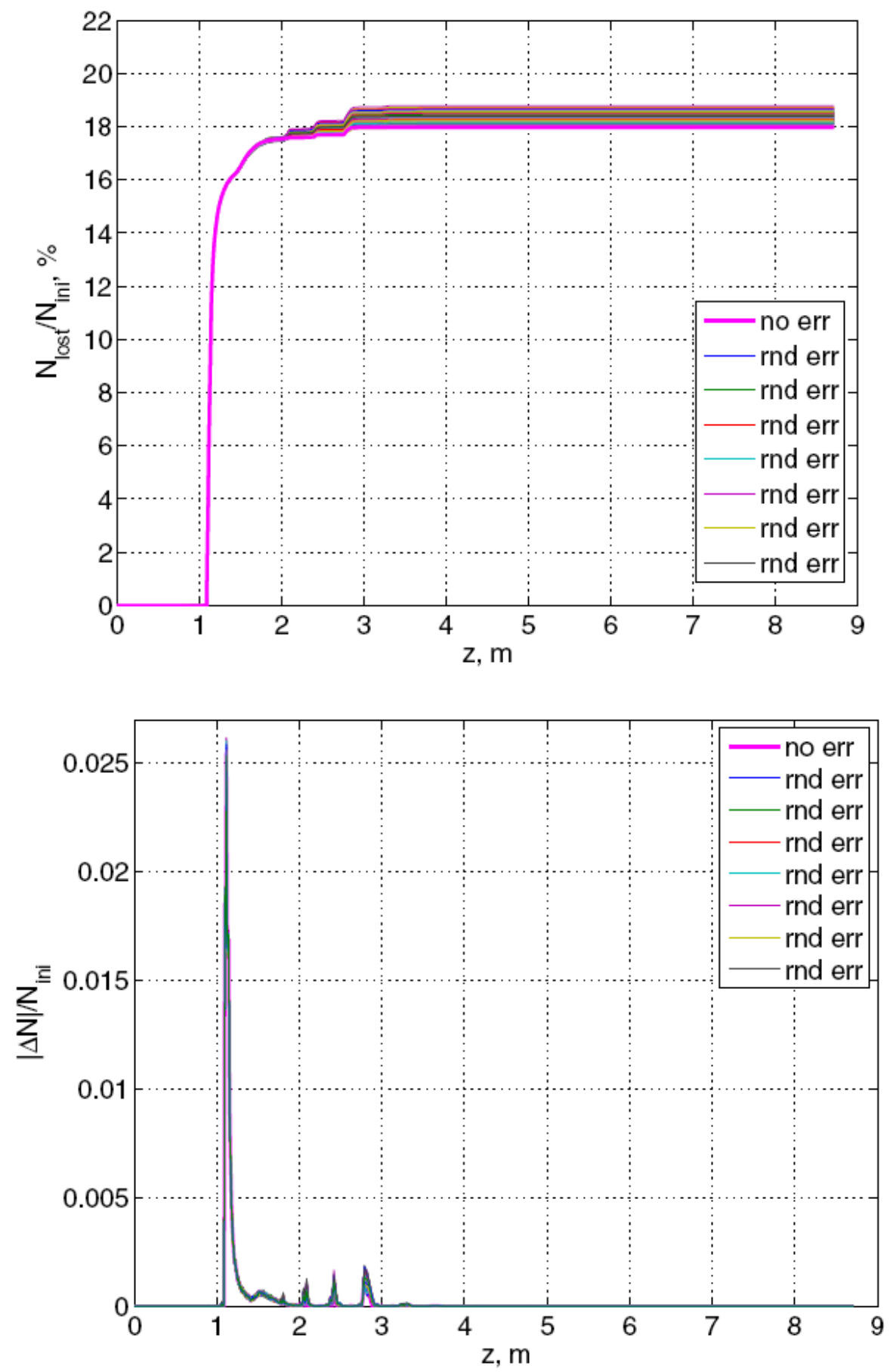

Fig. 3-4: MSU lattice: Beam losses versus distance along the beam line in meters with errors and steering. 

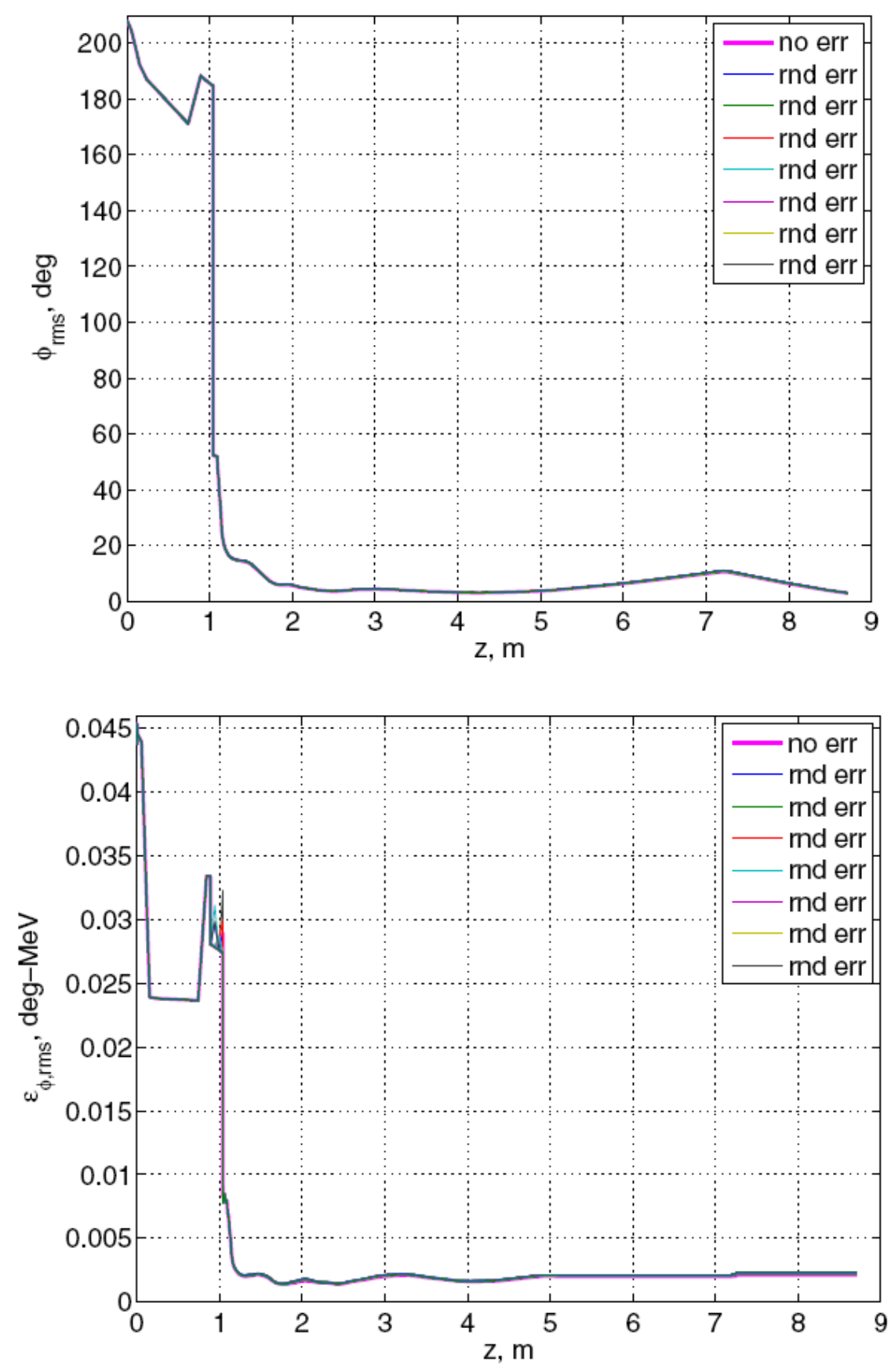

Fig. 3-5: MSU lattice: Beam longitudinal rms phase size (top) and longitudinal rms emittance (bottom) distance along the beam line in meters with (100 lines, cycling colors) and without errors (thick, magenta). 


\subsection{Results of 100 RIAPMTQ runs for the ANL design example}

Pictures below compare results for the ANL configuration from 100 runs in RIAPMTQ with random errors and steering with the results from a run without errors or steering, all with $200 \mathrm{~K}$ particles.

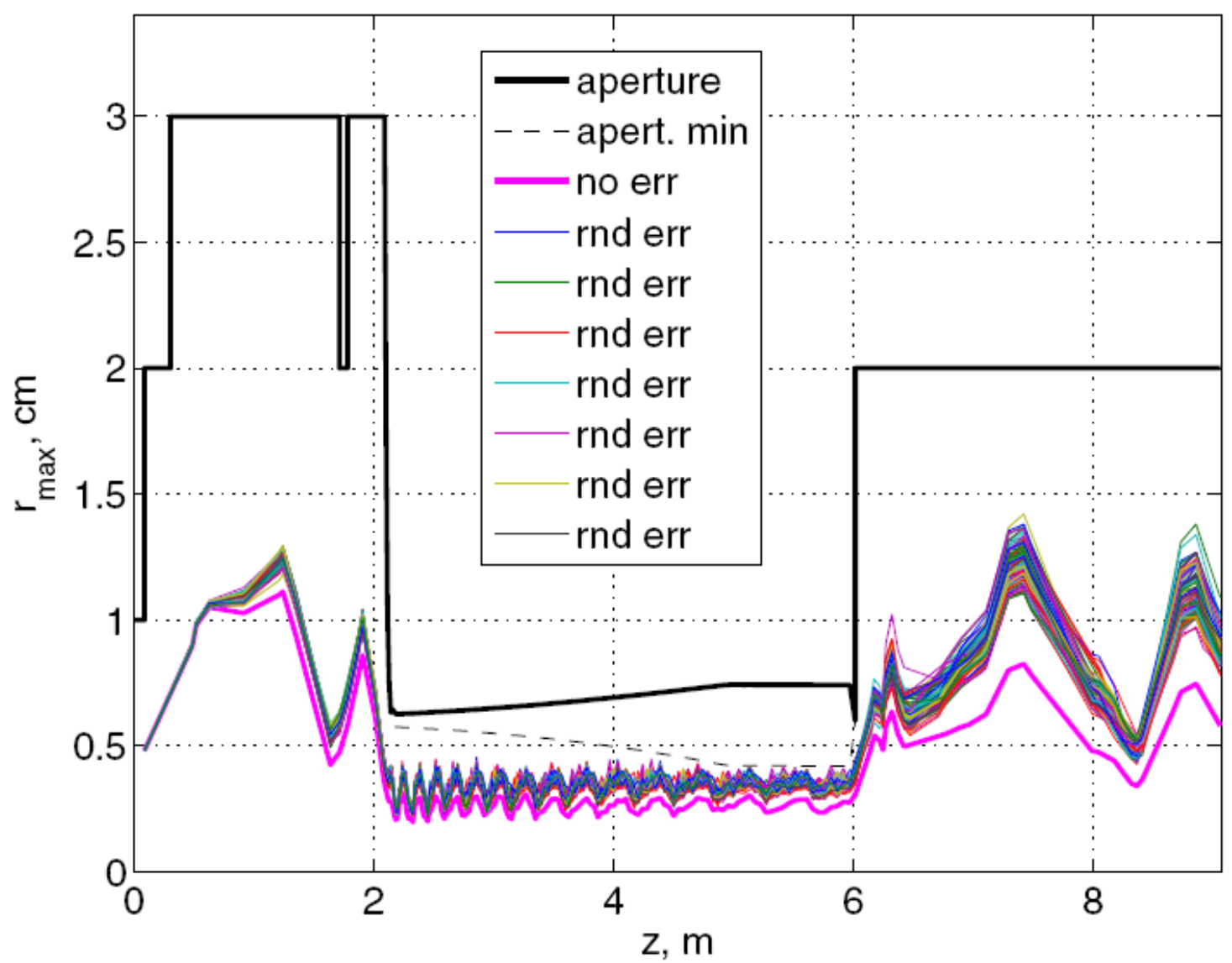

Fig. 4-1: ANL lattice: Beam maximal radius versus distance along the beam line in meters without random errors and steering (thick line, magenta) and with random errors and steering, 100 lines (cycling colors) where each line corresponds to a given run. The aperture is also shown in black. 

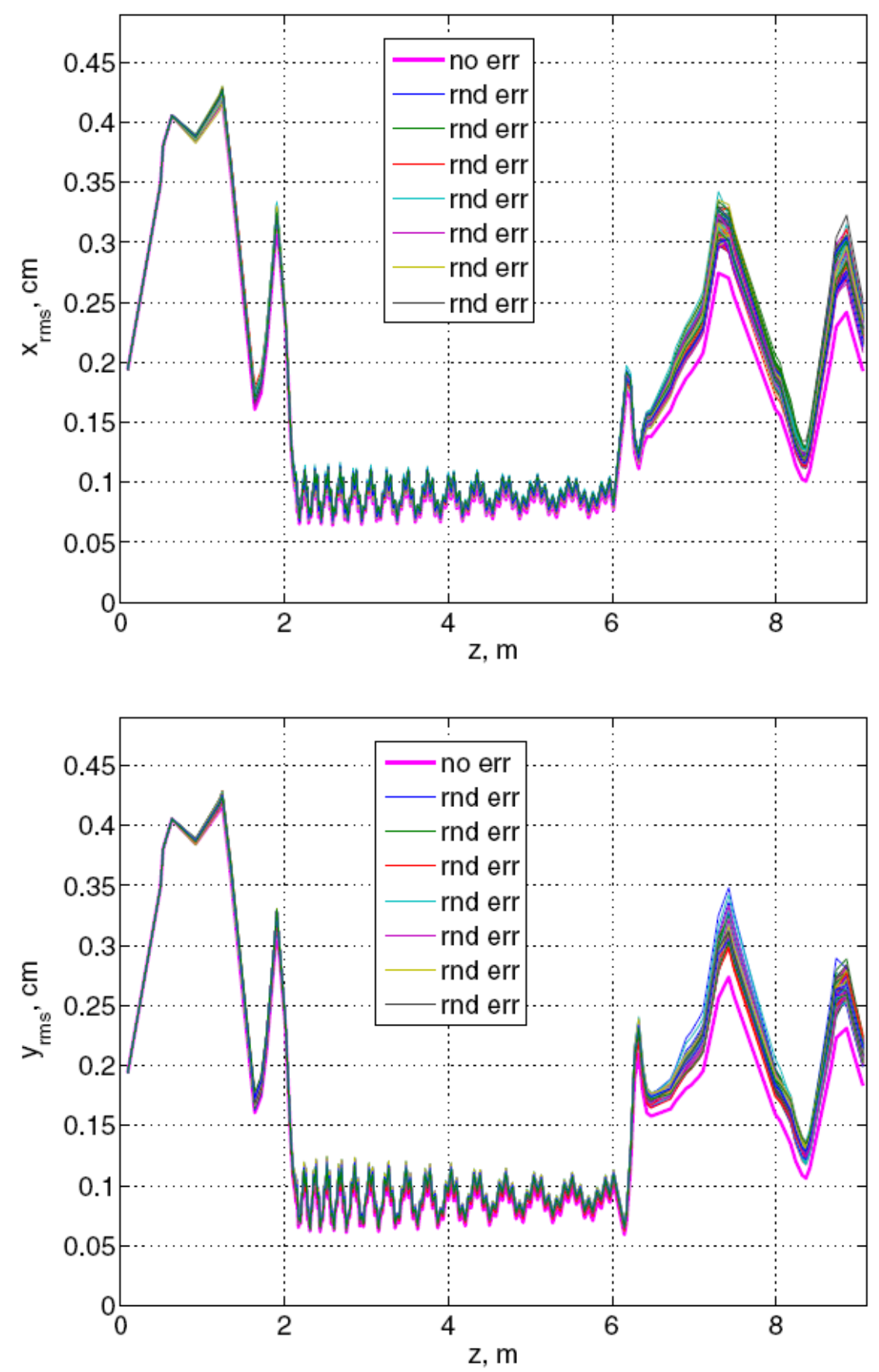

Fig. 4-2: ANL lattice: Beam transverse rms size with (100 curves, cycling colors) and without errors (thick line, magenta). 

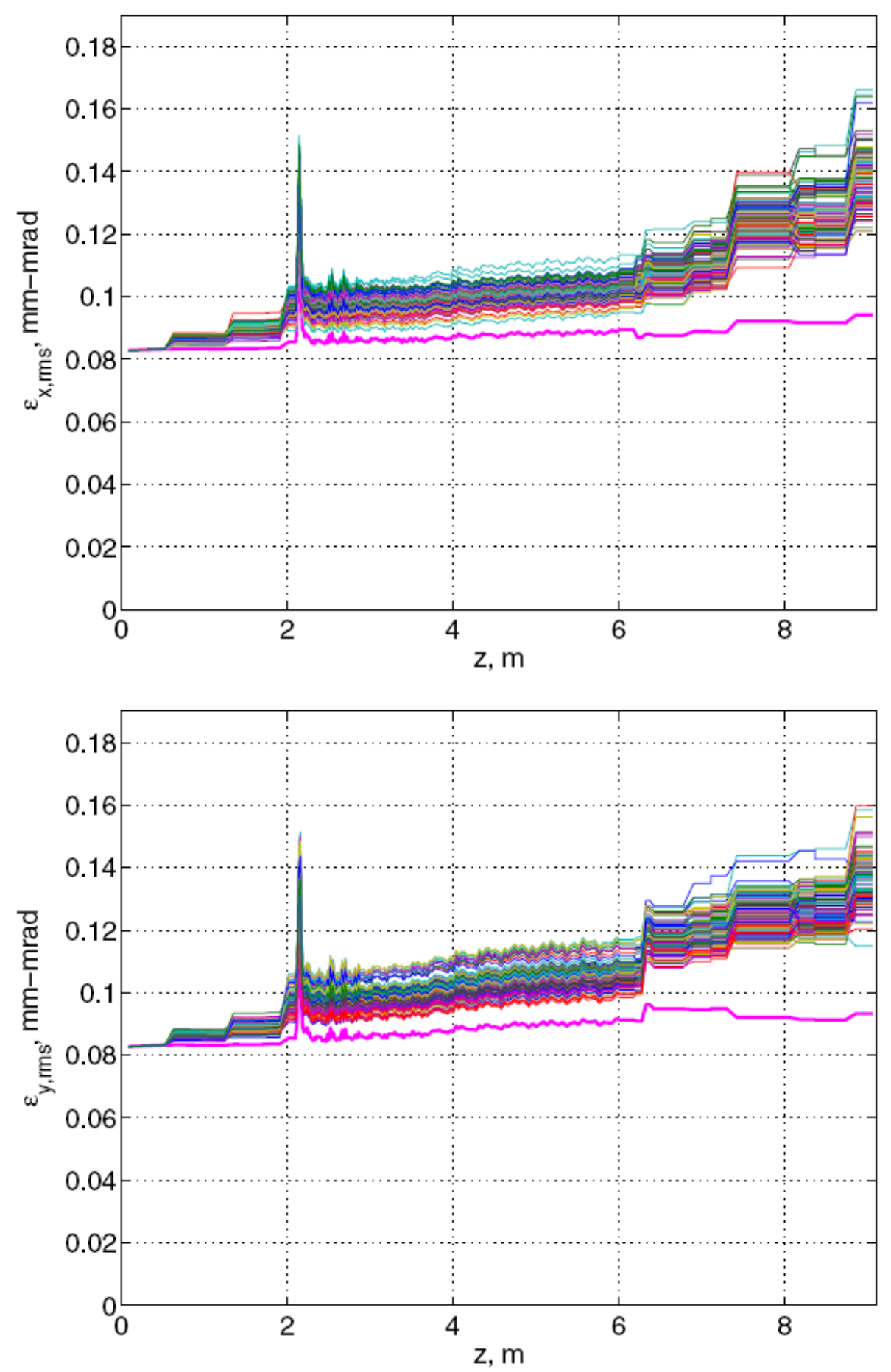

Fig. 4-3: ANL lattice: Beam transverse rms emittance with (100 lines, cycling colors) and without errors (thick, magenta). 

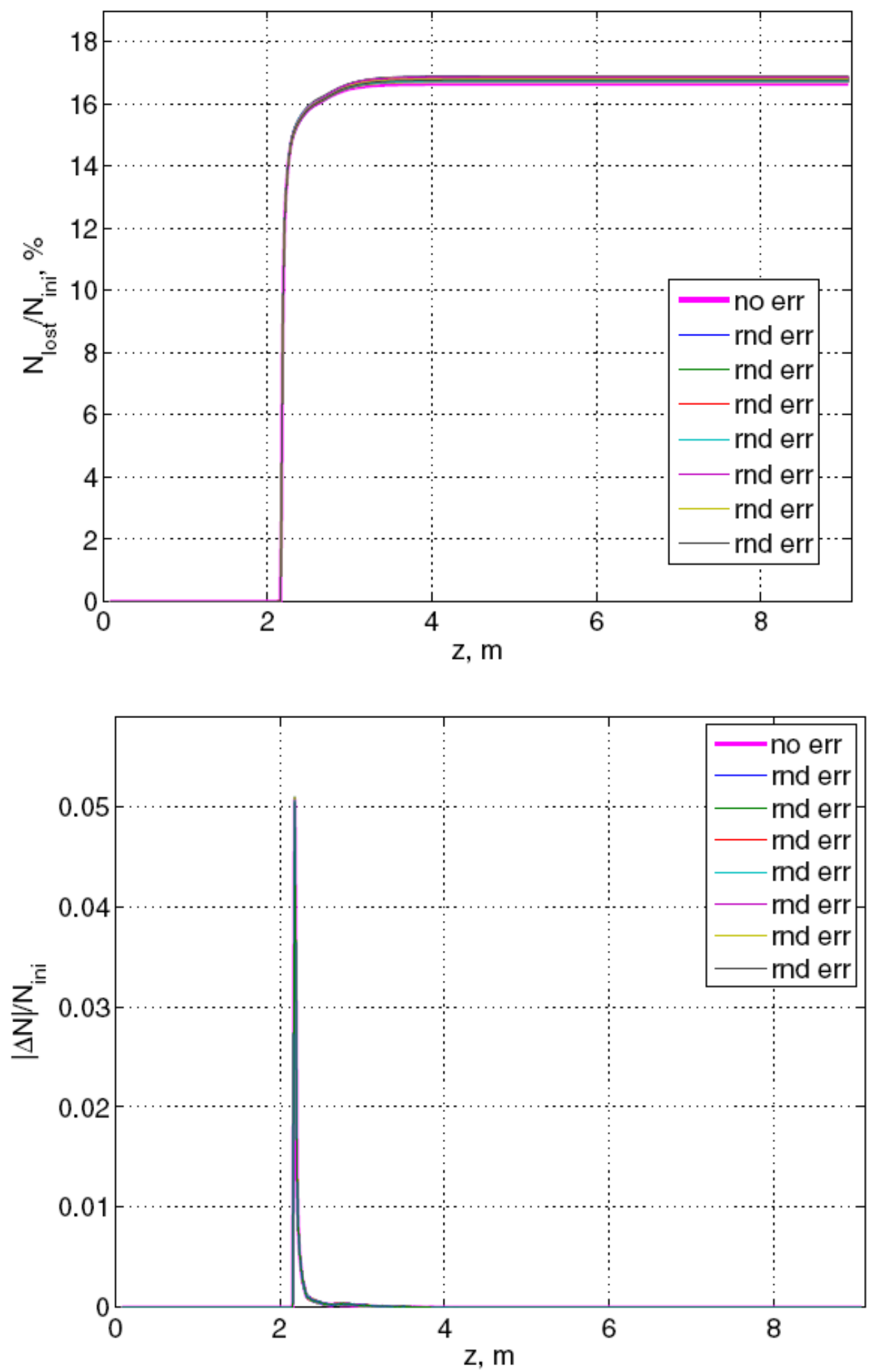

Fig. 4-4: ANL lattice: Beam losses versus distance along beam line in meters with (100 lines, cycling colors) and without errors (thick, magenta). 

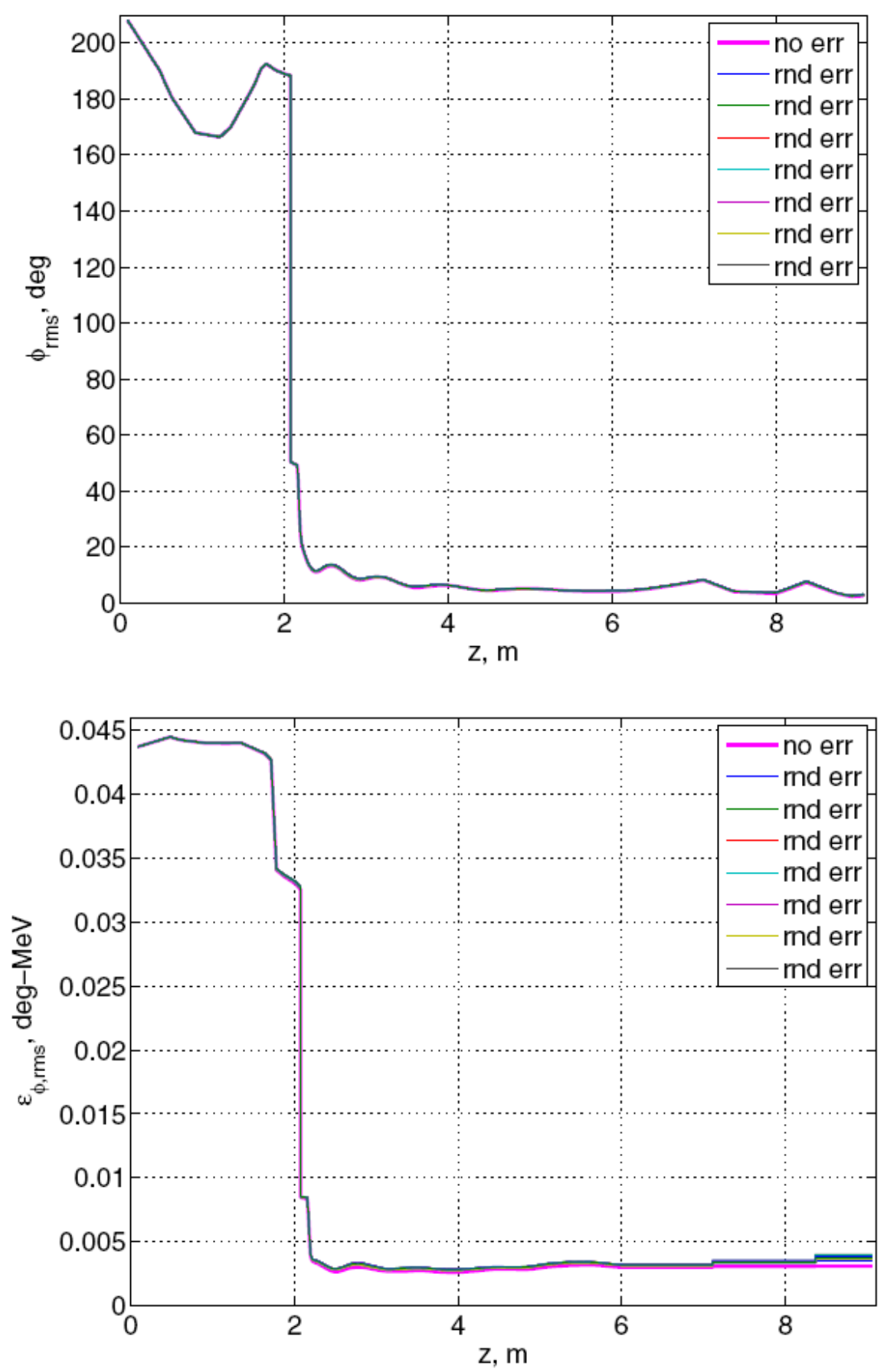

Fig. 4-5: ANL lattice: Beam longitudinal rms size (top) and emittance (bottom) with (100 lines, cycling colors) and without errors (thick, magenta). 


\subsection{Results of 100 IMPACT runs for the MSU design example}

Pictures below compare results for an MSU lattice from 100 runs with random errors and steering with the results from a run without errors or steering, all with $200 \mathrm{~K}$ particles.

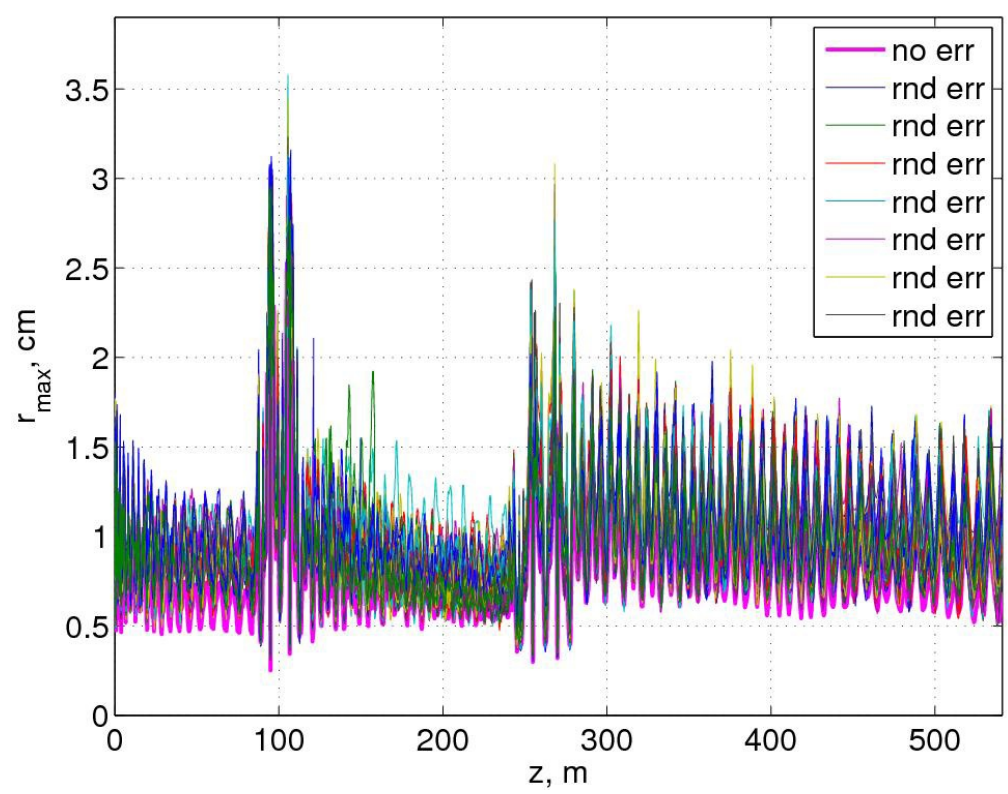

Fig. 5-1: MSU lattice: Beam maximal radius versus distance along the beam line in meters without random errors and steering (thick line, magenta) and with random errors and steering, 100 lines (cycling colors) where each line corresponds to a given run. The aperture radius varies from $1.5 \mathrm{~cm}$ to $4.0 \mathrm{~cm}$.

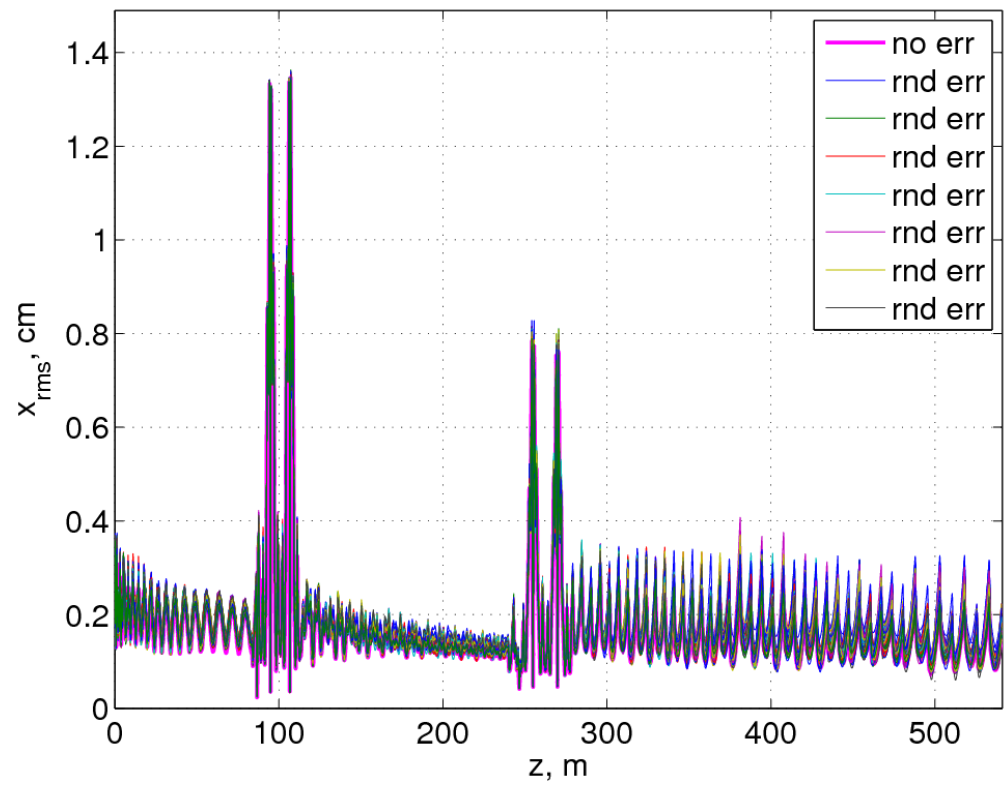




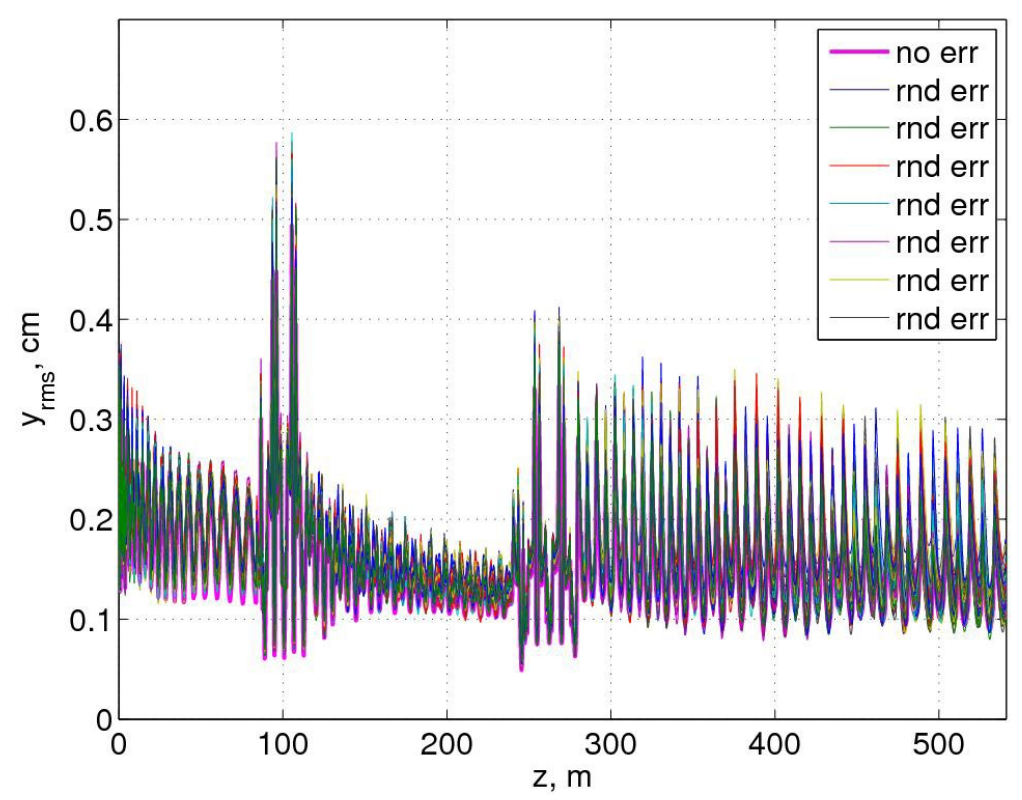

Fig. 5-2: MSU lattice: Beam rms sizes in $\mathrm{x}$ and $\mathrm{y}$ versus distance along the beam line in meters without random errors and steering (thick line, magenta) and with random errors and steering, 100 lines (cycling colors) where each line corresponds to a given run.

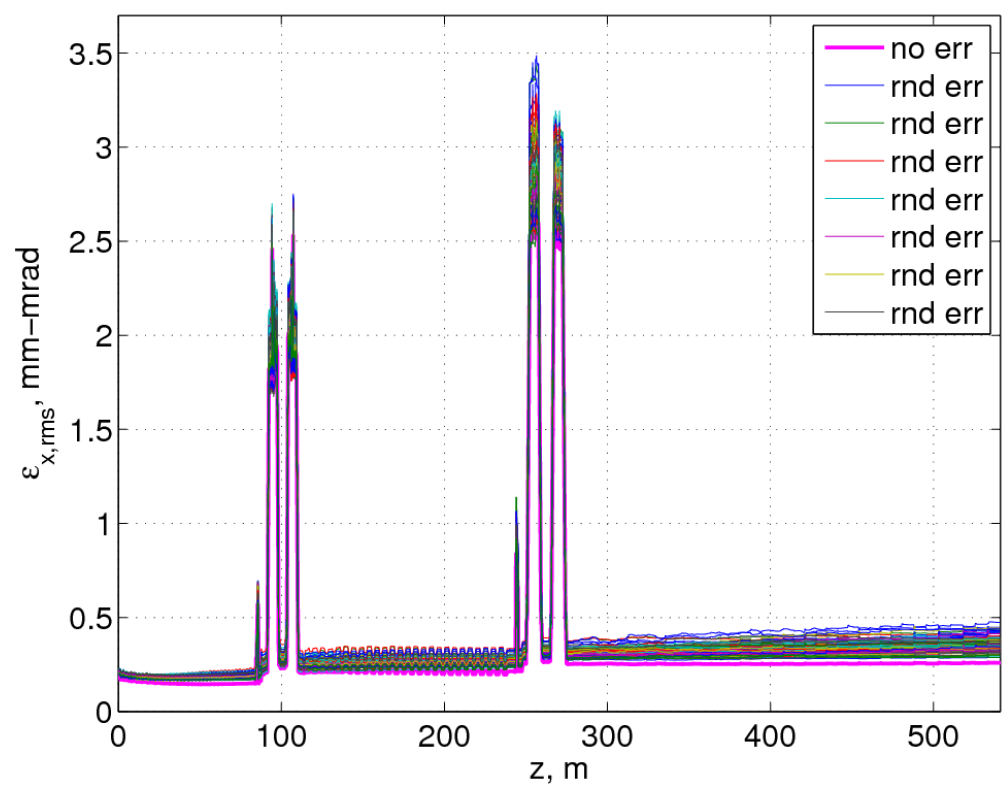




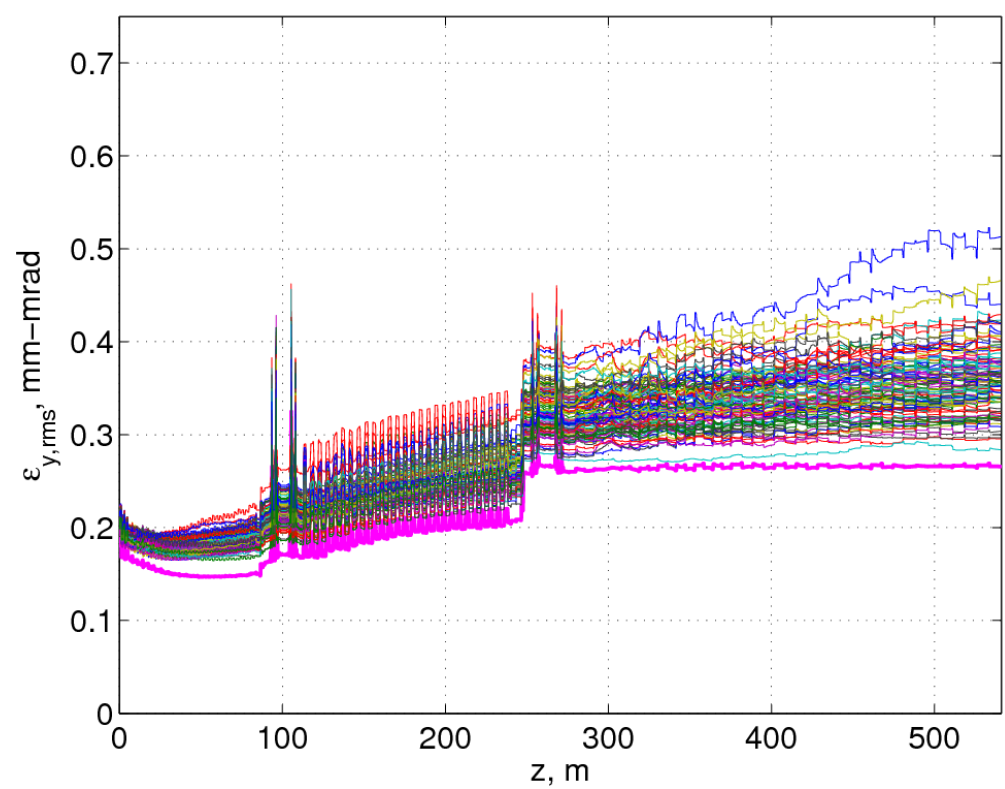

Fig. 5-3: MSU lattice: Beam rms emittances in $\mathrm{x}$ and $\mathrm{y}$ versus distance along the beam line in meters without random errors and steering (thick line, magenta) and with random errors and steering, 100 lines (cycling colors) where each line corresponds to a given run.

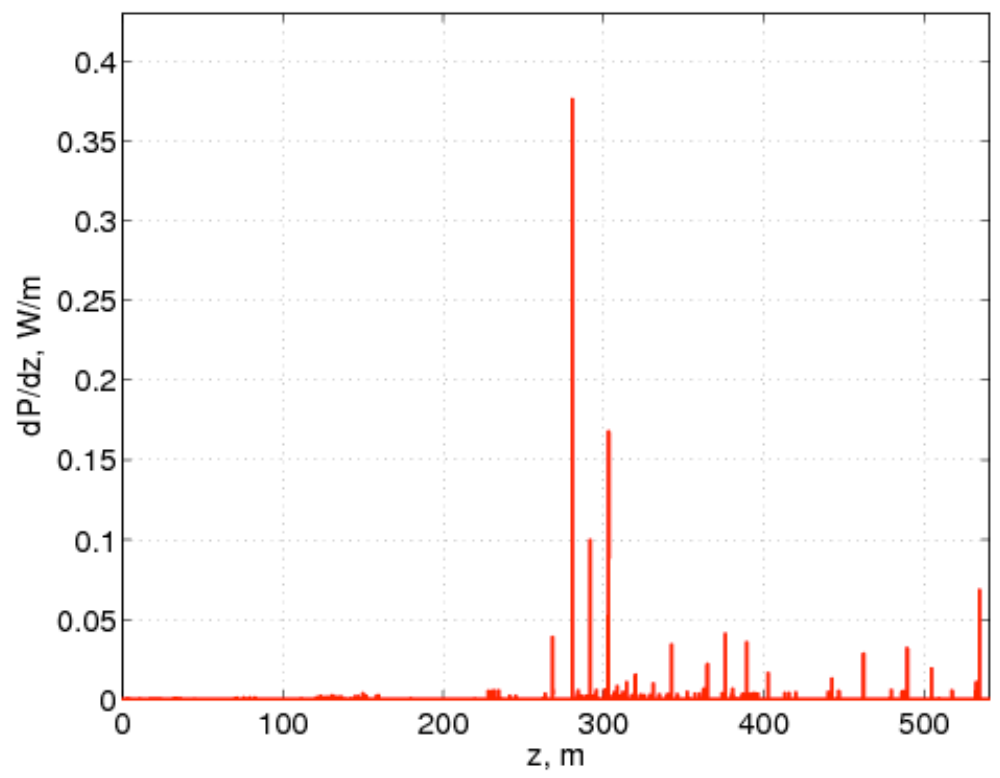

Fig. 5-4: MSU lattice: Beam losses in Watts per meter versus distance along the beam line in meters with errors and steering. 


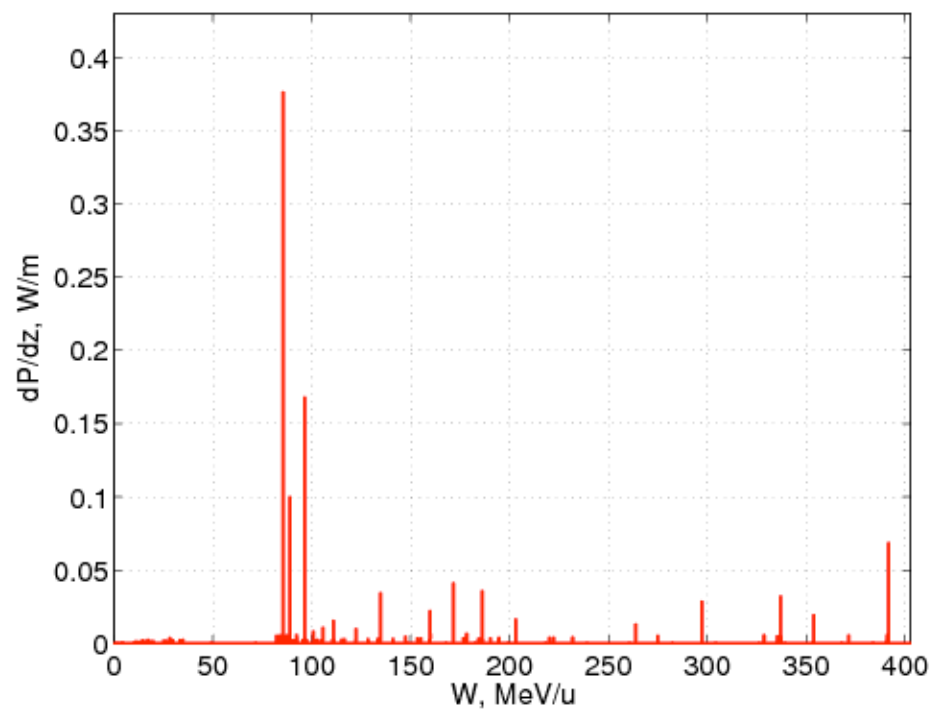

Fig. 5-5: MSU lattice: Beam losses versus kinetic energy with (100 lines, cycling colors) and without errors (thick, magenta).

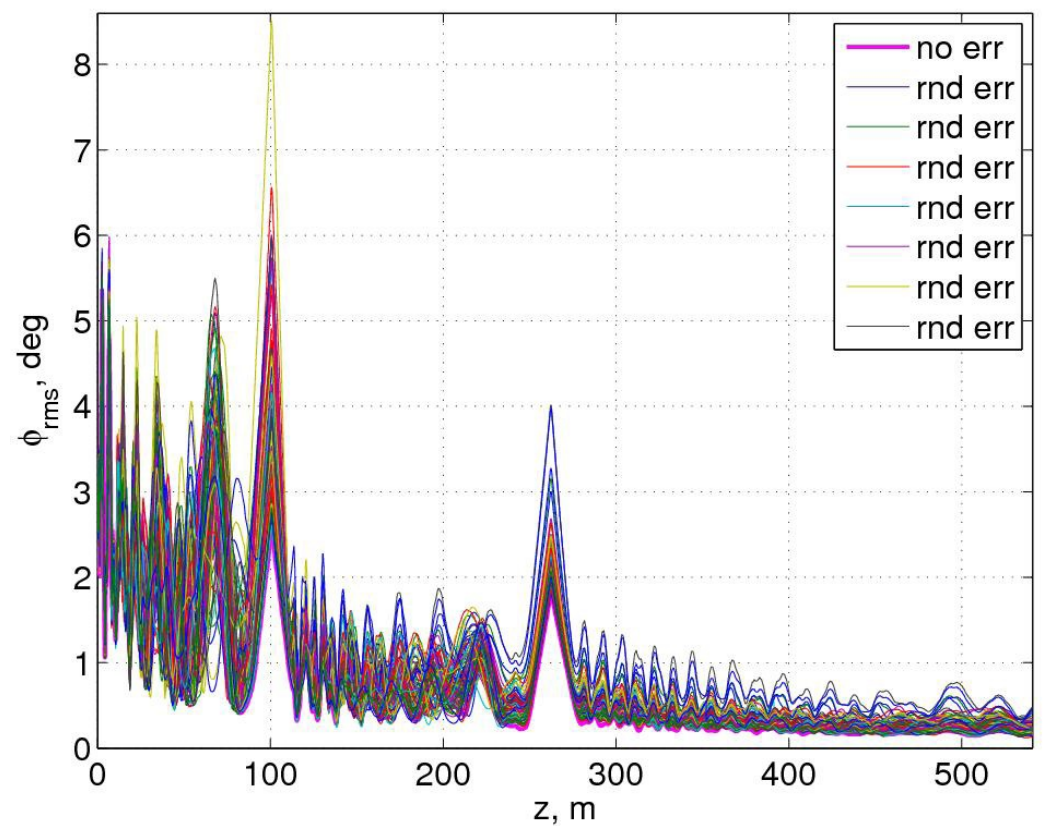




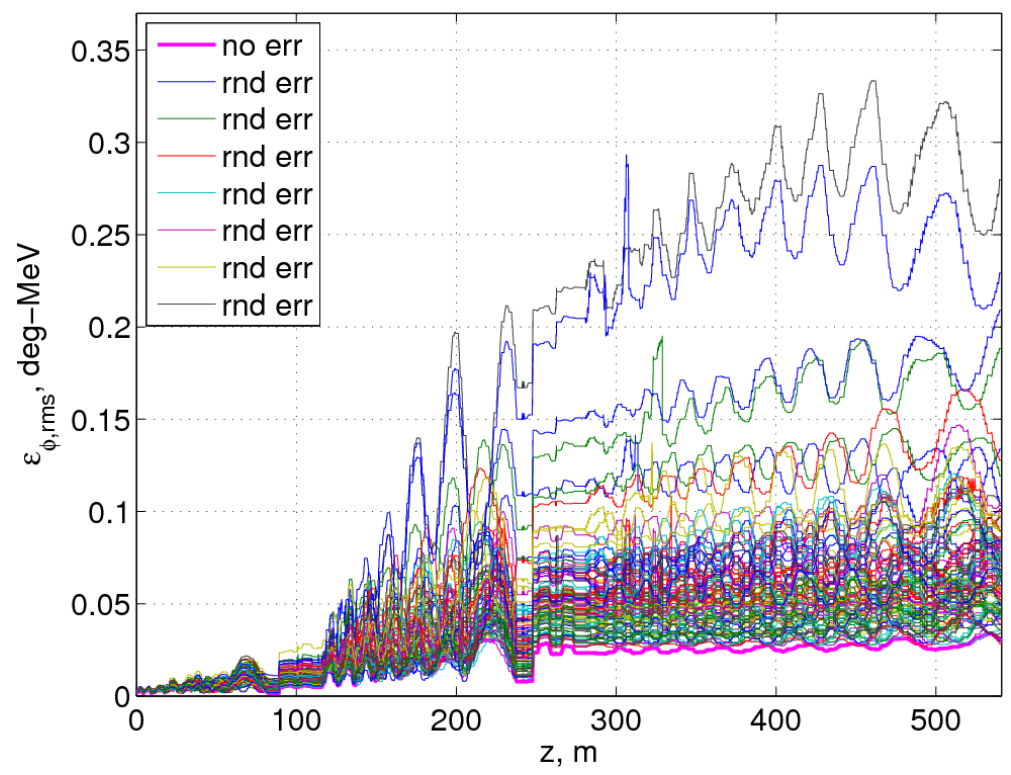

Fig. 5-6: MSU lattice: Beam longitudinal rms phase size (top) and longitudinal rms emittance (bottom) distance along the beam line in meters with (100 lines, cycling colors) and without errors (thick, magenta).

\subsection{Results of 100 IMPACT runs for the ANL design example}

Pictures below compare results for the ANL configuration from 100 runs with random errors and steering with the results from a run without errors or steering, all with $200 \mathrm{~K}$ particles.

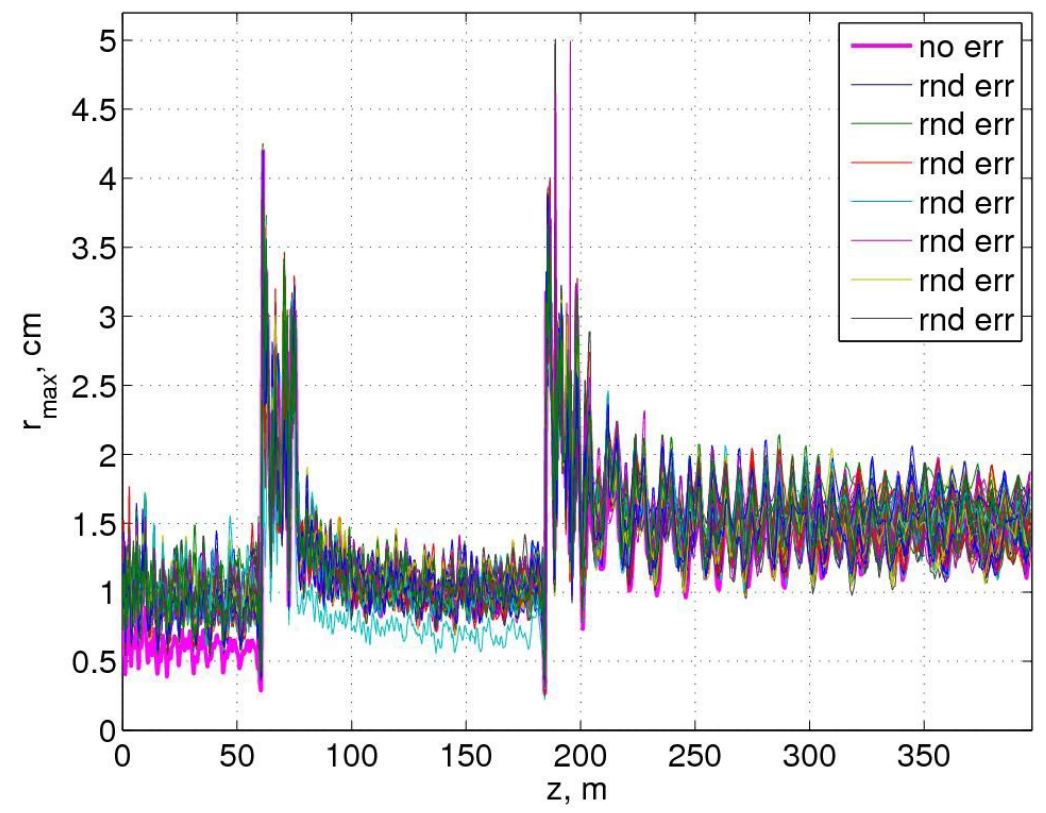

Fig. 6-1: ANL lattice: Beam maximal radius without errors (thick line, magenta) and with random errors (100 lines, cycling colors). The aperture radius varies from $1.5 \mathrm{~cm}$ to $4.0 \mathrm{~cm}$. 

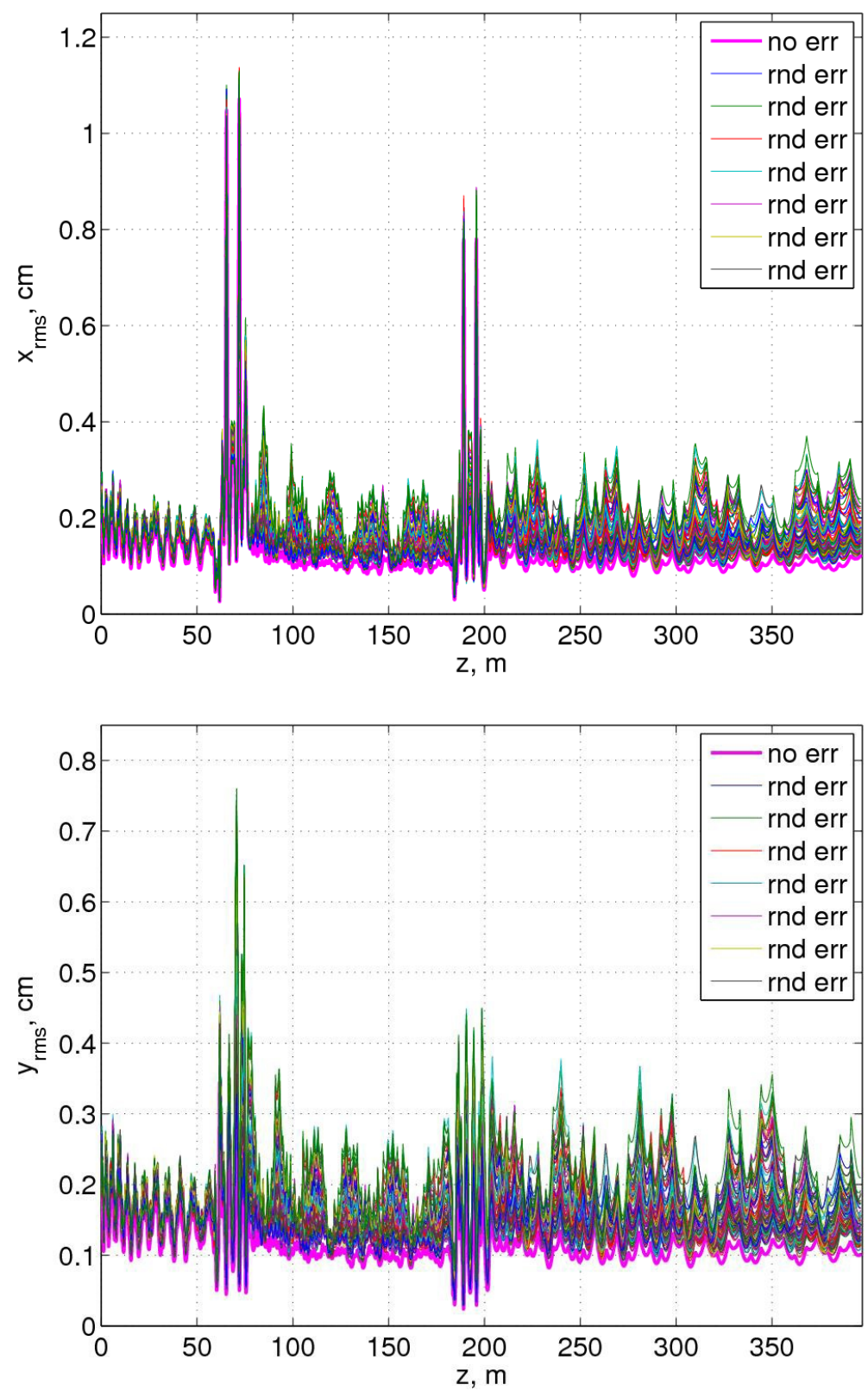

Fig. 6-2: ANL lattice: Beam transverse rms size with (100 curves, cycling colors) and without errors (thick line, magenta). 

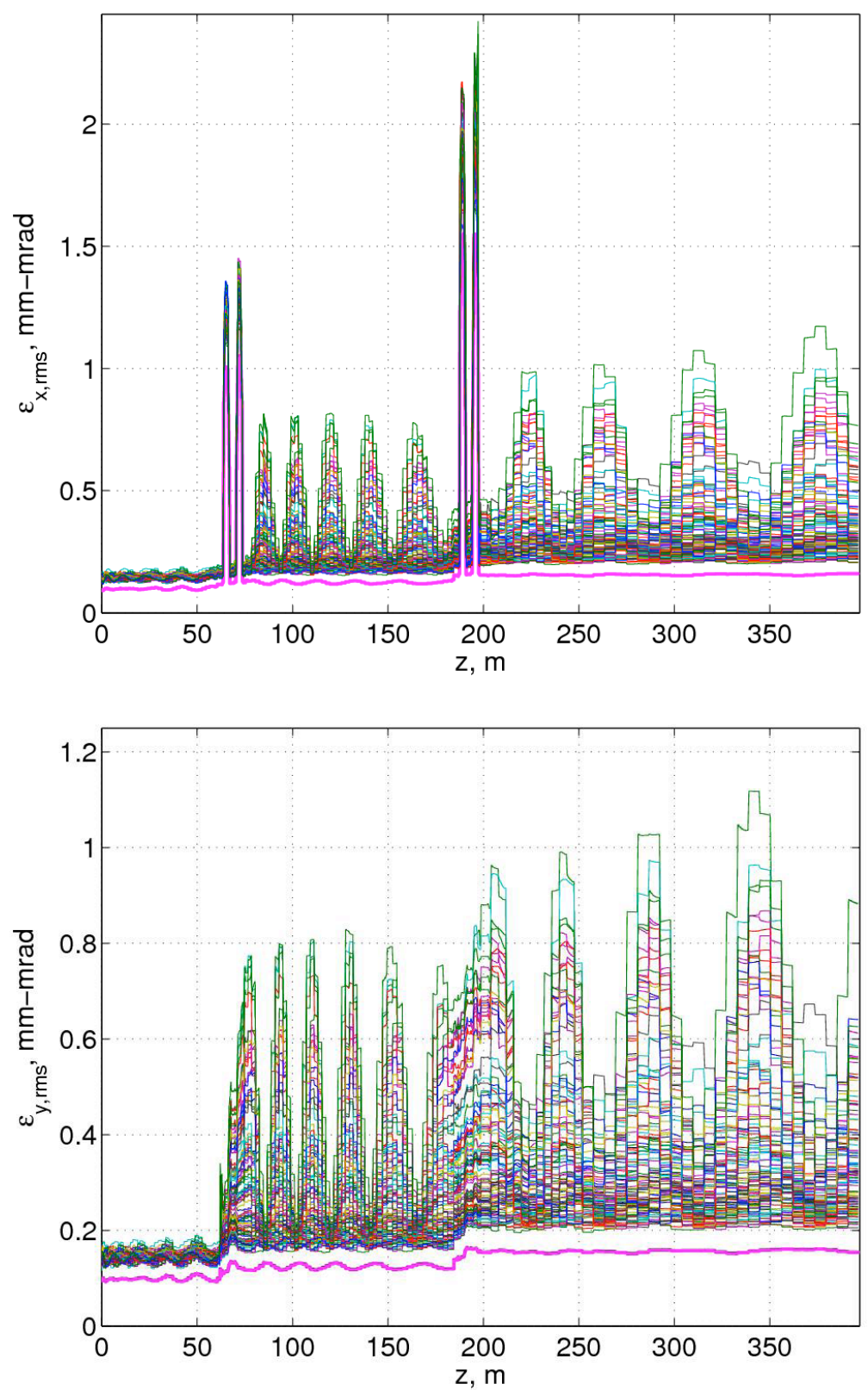

Fig. 6-3: ANL lattice: Beam transverse rms emittance with (100 lines, cycling colors) and without errors (thick, magenta). 


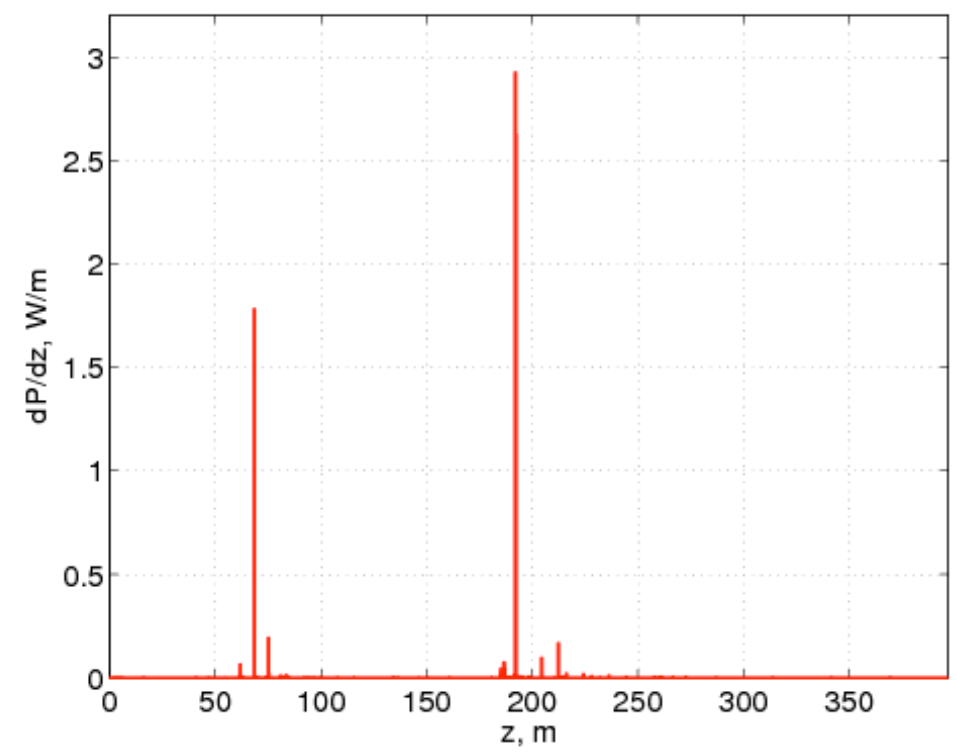

Fig. 6-4: ANL lattice: Beam losses versus distance along beam line in meters with (100 lines, cycling colors) and without errors (thick, magenta).

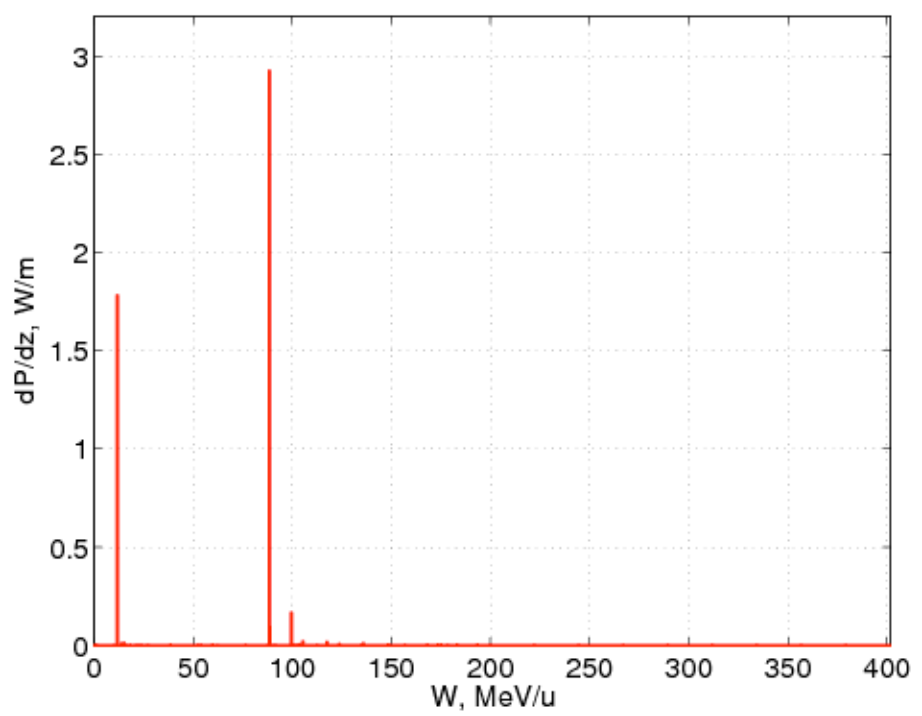

Fig. 6-5: ANL lattice: Beam losses versus kinetic energy with (100 lines, cycling colors) and without errors (thick, magenta). 

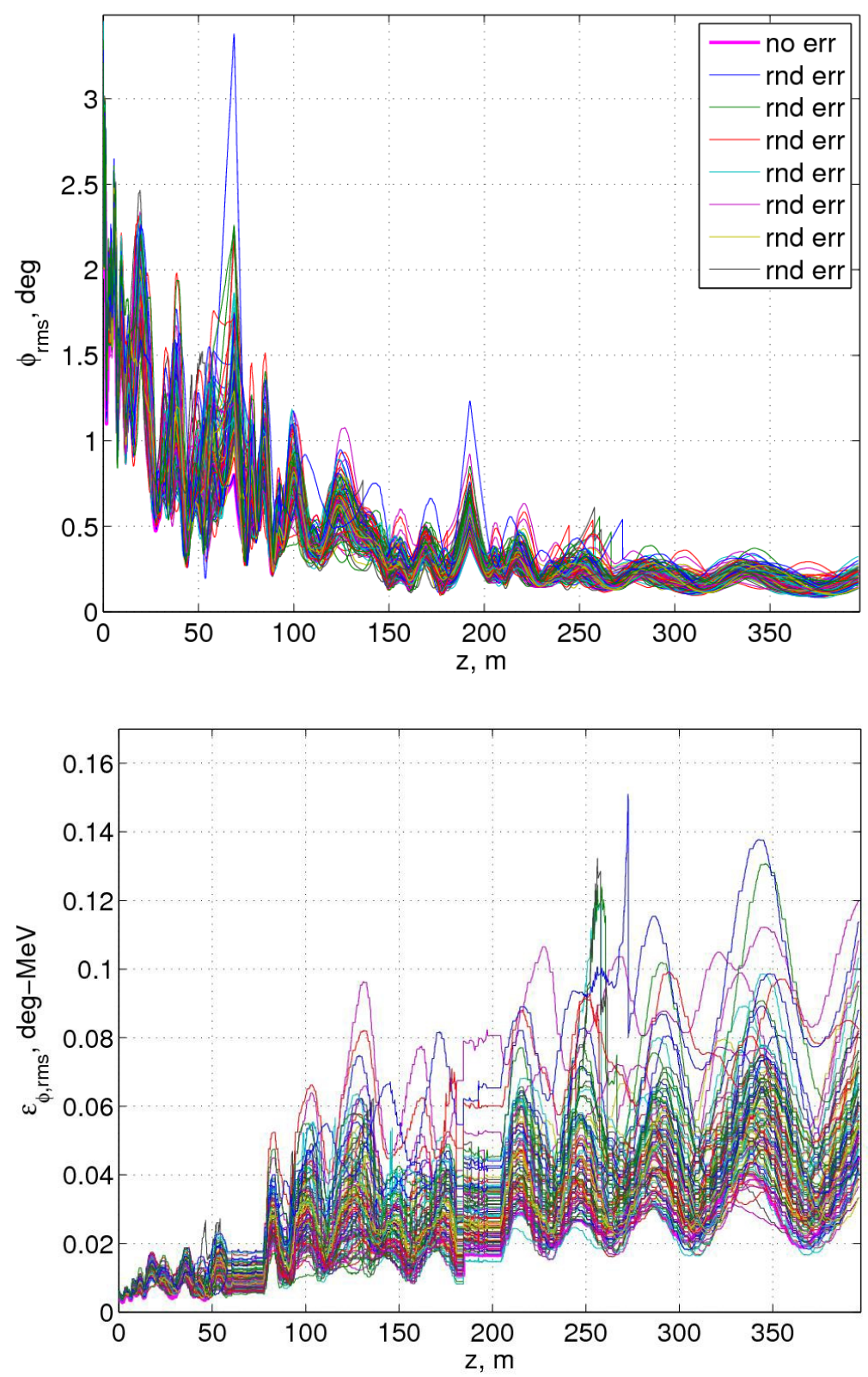

Fig. 6-6: ANL lattice: Beam longitudinal rms size (top) and emittance (bottom) with (100 lines, cycling colors) and without errors (thick, magenta).

\subsection{Beam Loss Predictions/Summary}

The RIAPMTQ code calculates the low-energy beam losses. The plots are shown in Fig. 3.4 (top and bottom) for the MSU design example, and in Fig. 4.4 (top and bottom) for the ANL design example. The beam transmission through the RFQ is about $83 \%$ for both designs. This is a good RFQ transmission when the bunching is done with a multiharmonic buncher; the corresponding losses in and after the RFQ at this low energy are not of concern for radioactivation. 
The beam losses in main linac, predicted by the code IMPACT, are the ones that motivated our study. The fractional beam losses in the main linac for the two design examples were in the range of $10^{-4}$. Concern about activation has resulted in a conservative criterion that it is desirable to limit the high-energy beam losses to values equivalent to activation caused by 1 Watt per meter loss at $400 \mathrm{MeV} / \mathrm{u}$, based on activation that scales approximately as lost-particle kinetic-energy squared. These Watt per meter values are calculated in the simulations by adding the energies of the lost particles in each element, and dividing by the length of that element.

The results for the MSU design example are shown in Fig. 5.4 (Watts per meter versus distance), and Fig. 5.5 (Watts per meter versus kinetic energy per amu). The 100 runs with $200 \mathrm{~K}$ particles per run for the MSU RIA design example, using the error tolerances given in Section 1, gave a prediction that the largest particle losses in the main linac are less than $0.4 \mathrm{~W} / \mathrm{m}$, and the typical losses are more than an order of magnitude smaller. The largest loss value ( 0.4 Watts per meter) occurs at about $85 \mathrm{MeV} / \mathrm{u}$. This result is well below an activation-equivalent beam loss criterion of 22 Watts per meter at $85 \mathrm{MeV}$, corresponding to $1 \mathrm{~W}$ per meter at $400 \mathrm{MeV} / \mathrm{u}$. This loss peak in the RIA design occurred after the chicane and at a superconducting cavity. The results for the high-energy beam losses above $100 \mathrm{MeV}$, when machine errors are included, are nearly all well below 0.05 $\mathrm{W} / \mathrm{m}$. If desired, it should be feasible to reduce of this peak by installing collimators in the room-temperature chicane just upstream of where the loss occurs.

The beam-loss results for the ANL design example are shown in Fig. 6.4 (Watts per meter versus distance), and Fig. 6.5 (Watts per meter versus kinetic energy per amu). Two beam-loss peaks are observed, one about $10 \mathrm{MeV} / \mathrm{u}$, and the other at about 90 $\mathrm{MeV} / \mathrm{u}$, where the predicted loss is about 2.9 Watts per meter. These beam loss peaks occur at superconducting RF buncher cavities, located after the chicane, the same as for the MSU design. The aperture radius where the peak losses occurred is $1.5 \mathrm{~cm}$, which is smaller than the nearby element apertures of $3.0 \mathrm{~cm}$. In the case of the ANL design example there are almost zero losses predicted for energies above $100 \mathrm{MeV} / \mathrm{u}$. From the viewpoint of activation, a loss of 2.9 Watts per meter at $90 \mathrm{MeV} / \mathrm{u}$ is not serious because at $90 \mathrm{MeV}$ the activation-equivalent loss criterion, corresponding to 1 Watt per meter loss at $400 \mathrm{MeV} / \mathrm{m}$, is about $20 \mathrm{Watts}$ per meter. The loss in the peak is a factor of 7 below this criterion. As was the case for the MSU design it should be feasible to reduce of this peak, if desired, by installing collimators in the room-temperature chicane just upstream of where the loss occurs.

In summary, the RIAPMTQ/IMPACT code package has been applied to the problem of estimating the beam losses in both design examples provided by ANL and MSU. Neither of these design examples was final RIA design, but represented an intermediate stage in the design process, and both were expected to be representative of good designs with low beam losses. The result is that, using the error tolerances given in Section 1, both designs meet an activation-equivalent beam-loss criterion of less than 1 Watt per meter. Collimation has not been included in either of these designs, and collimation could be used to reduce significantly the observed beam loss peaks seen below $100 \mathrm{MeV}$ in both design 
examples. Our funding allocation was sufficient to allow us to perform a study with only one set of beam-error tolerances. Additional studies could be made to evaluate the effects of other choices of the error tolerances and to evaluate the effects of collimation, but these should be carried out on the new linac designs for the planned radioactive beam facility. The main objective of our work, to apply the RIAPMTQ/IMPACT beamdynamics simulation package to confirm the low beam-loss expectation of these design examples, has been accomplished.

\title{
APPENDIX A
}

\section{Procedure for Automatic RIA Linac Error Studies Using the IMPACT Code}

\author{
Ji Qiang, Nov. 5, 2007, LBNL
}

This note summarizes an automatic procedure to simulate the RIA linac machine random errors using the parallel beam dynamics code, IMPACT. Two computer programs based on the python scripting language are developed to automatically generate the random errors and to calculate steering strength. The following gives a detailed description for this procedure.

This procedure includes the following four steps:

1) Generate machine random imperfection for solenoids, rf cavities, and quadrupoles in the linac.

2) Set up steering magnets and BPMs.

3) Find the steering strength for each pair of steering magnets through the whole 500 meter long linac.

4) Run the parallel IMPACT simulation

To generate an input lattice containing all the machine errors, we have developed a computer program, "scanerrorRIA.py" based on Python scripting language. This program reads in the input lattice file of the IMPACT without machine errors. Then it loops through all beam line elements. For each beam line element inside the range of the interest linac section, random errors for both end displacements, rotation, amplitude, and phase are added to the solenoid, quadrupole, and rf cavity element. The random errors are generated from a uniform distribution with the given maximum amplitudes. After the setting of random errors, the program creates a working directory for each set of random errors. Then, the IMPACT executable for multi-particle simulation, and for steering are copied into the working directory from a directory above. The input lattice file without the machine imperfections and the linear solver executable are also copied into the working directory. The script for parallel job submission is read in and modified to account for different random set. The stripper foil input files are also copied into the working directory. The particle output distribution at the end of the RFQ from the RIAPMTQ simulation is also copied into the working directory. To set up the input file for the IMPACT simulation, we need to know the charge states and the number of 
particles for each charge state. This information is extracted out from the particle distribution file and written into the IMPACT input file "test.in".

Given the input lattice file with random machine errors, the steering magnets and BPMs are inserted into the input file. The locations of steering magnets and the BPMs have strong impact on the beam centroid oscillation amplitude with steering. We have tried different schemes such as using one steering magnet and BPM after each cryo-module and found a large centroid oscillation and particle losses for this scheme. Putting steering magnet in front of the solenoid also results in larger centroid orbit oscillation. In the current version of the error steering, we assume that there is steering magnet after each solenoid and quadrupole, and a BPM after each cryo-module. An automatic computer program, scansteer.py using the Python script language is developed to create such input file and to calculate the steering strength. Since the steering strength is calculated based on using five particles and an averaged charge state 28.5 of U238 as the reference particle, the first 2 lines of input file "test.in" will be modified. This includes replacing the number of processor for parallel simulation with the single processor run, changing the number of particles in the simulation, changing the number of charge state to 1 , changing the charge over mass ratio, setting the initial distribution, etc. Next, the program loops through the lattice of the linac. For the first two section of linac, a steering magnet is inserted after each solenoid. For each pair of steering magnets, a restart function line is added after the second steering magnet. A BPM is inserted in the warm drift section, which is the middle of two superconducting cryomodules. To do this, the original drift line is broken into two drift lines, with the BPM line inserted between them. Following the BPM line, a particle distribution output line and a stopping line are added so that the code can be stopped right after the second BPM. The third section of MSU RIA linac consists of doublet quadrupole focusing and elliptical superconducting rf cavities. A steering magnet is inserted after each quadrupole. Again, the second quadrupole of each pair is attached by a restarting function line. A BPM is inserted after each superconducting cryomodule. Five additional BPMs are inserted in the specific location of the linac. The input files for the foil stripper are modified so that there is only one charge state, i.e. the reference charge state, after each stripper instead of five or three charge in real simulation.

The steering strength is calculated for each pair of steering magnets using the two BPMs down stream. Five particles are tracked with one particle starting on-axis. The other four particles have the same initial conditions as the first particle and receive a small kick in either horizontal or vertical direction at the location of steering magnet. The horizontal and vertical coordinate differences at the location of BPMs between those particles and the first particle are used to calculate the response matrix. In this study, we have assumed that all the beam elements are turned off from the second steering magnet down stream to the BPM locations. Using the response matrix and the horizontal and vertical coordinates of the first particle, the steering strength is obtained by solving the following equation:

$$
\mathrm{R} \mathrm{S}+\mathrm{B}=0
$$

Here, $\mathrm{R}$ is the $4 \times 4$ response matrix, $\mathrm{S}$ is the steering strength vector and $\mathrm{B}$ is the coordinates of the first particle at the locations of two BPMs. Another IMPACT simulation is done including the steering kicks at the location of steering magnets. The 
restart function line after the second steering magnet records the information such as particle distribution for next pair of steering strength calculation. This procedure is repeated for a number of pair of steering magnets till the end of linac. To calculate the steering strength, the computer program scansteer.py loops through the whole linac lattice first. The locations of steering magnets and BPMs are recorded and stored in two arrays. Then, the program loops through total pairs of steering magnets. For each pair of steering magnets, the program finds the two nearest BPMs down stream the steering magnets. Depending on the location of the steering magnets, the charge and the charge over mass ratio for the reference particle in the first 12 lines of the input file will be modified to account for the charge state change through the stripper foils. The output file names of particle distribution after the BPMs are also updated as 71 for the BPM1 and 72 for the BPM2. The focusing and accelerating beam line elements from the second steering magnet down stream to the second BPM are turned off. The stopping line after the second BPM is turned on. The IMPACT simulation is done for the five particles described above from the last restarting point (i.e. after the second steering magnet). The particle distribution output at two BPM locations are extracted from fort.71 and fort.72. This information is used to find the response matrix $\mathrm{R}$ and $\mathrm{B}$. A linear algebraic solver is used to solve the above equation using the Gaussian elimination. The solution for steering strength is saved in a temporary file fort.75 and read in. The obtained steering strength is then written into the input linac lattice file of the IMPACT code. Here, one flag of the steering magnet is switched from the original 2 (for initial small kick) to 0 (for applying steering kick). Using the updated steering strength, another IMPACT simulation is done for the five particle (with same initial conditions) to the stopping line. A restarting function point is set after the second steering magnet. This point will be used as the starting location for the next pair of steering strength calculation. We have used two lists to store the lattice information from the input file: the first one stores the updated steering strength only, the second one stores the modified lattice from the second steering magnet down to the second BPM. After the second IMPACT run for each pair of steering magnets, the second list is replaced by the first list. This procedure is repeated for a number of times so that the strength of all steering magnets is set. The restart switch flag in the first 13 lines is turned off. All restarting point lines and the particle distribution output line after the BPM are turned off. The first 13 lines in the original input file "test.in" are copied into the new "test.in" with all steering strength set. The input files for the striper foils are copied back for multiple charge state.

An IMPACT parallel simulation is submitted to the queue from the working directory and back to one directory above. A new working directory is built and the above three steps are repeated for that new working directory. This loop will stop when the given number of random error set is reached.

The following are some sample results from above procedure.

Fig. 1 shows the horizontal centroid amplitude with and without steering. 


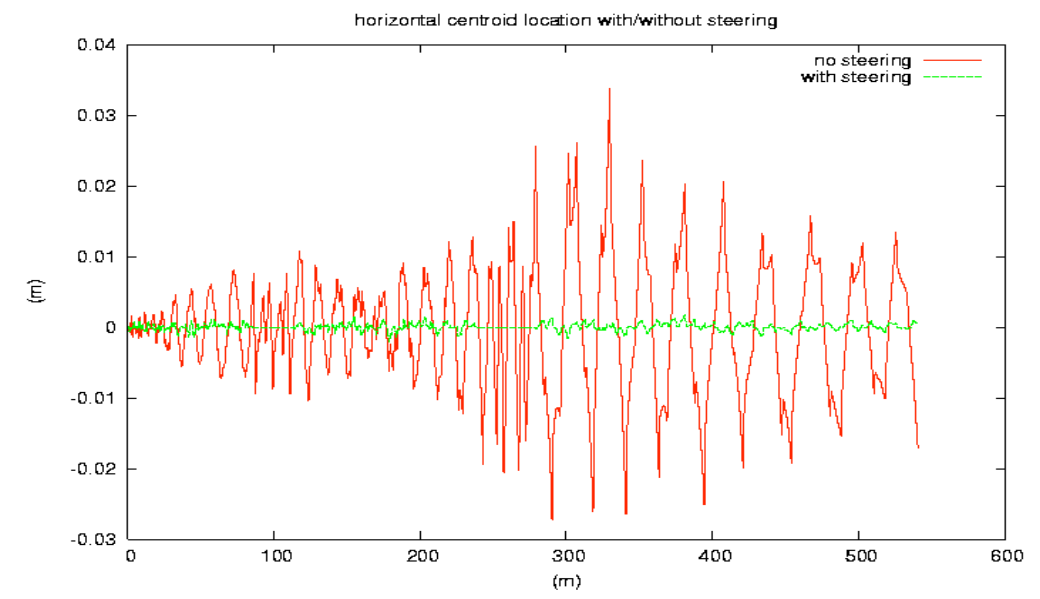

Fig. 2 shows the particle survive through the MSU RIA linac with and without automatic steering for one machine random seed.

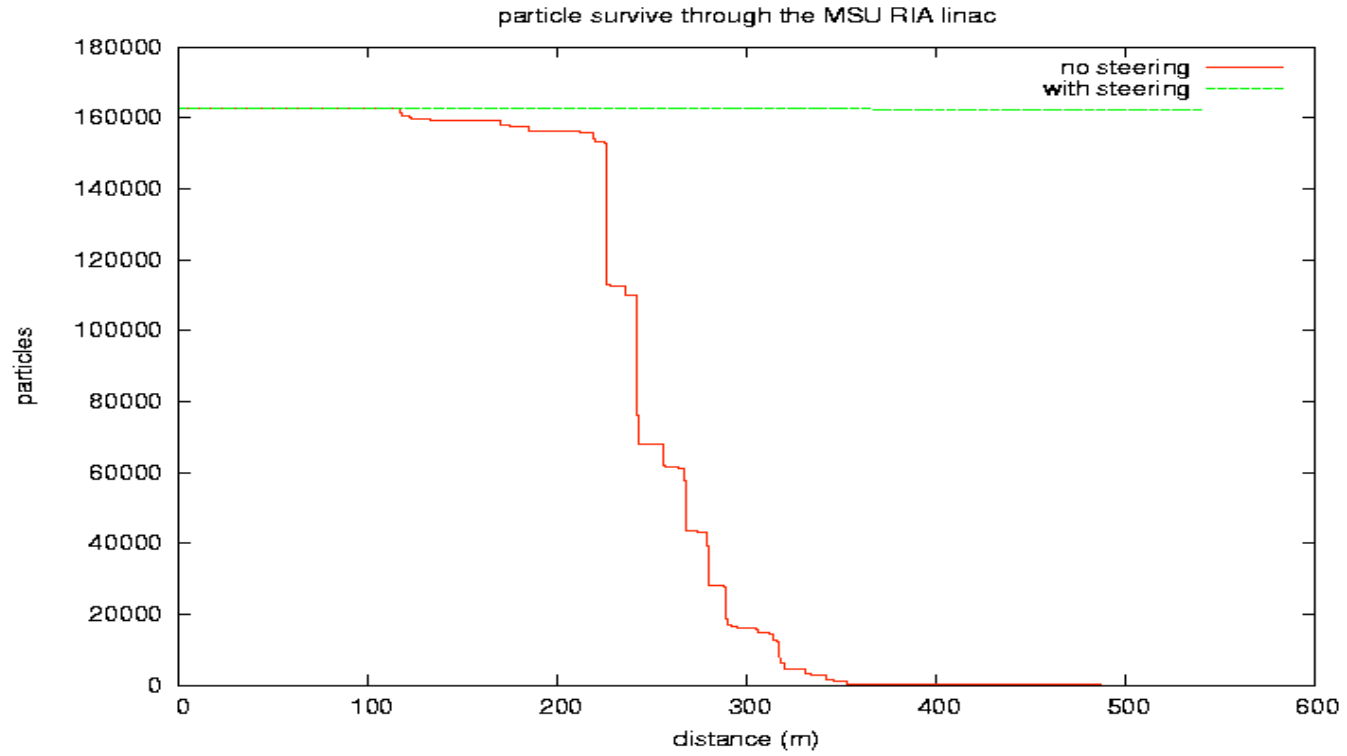

Fig. 3 shows the particle survive through the MSU RIA linac with automatic steering and 10 random machine errors. 


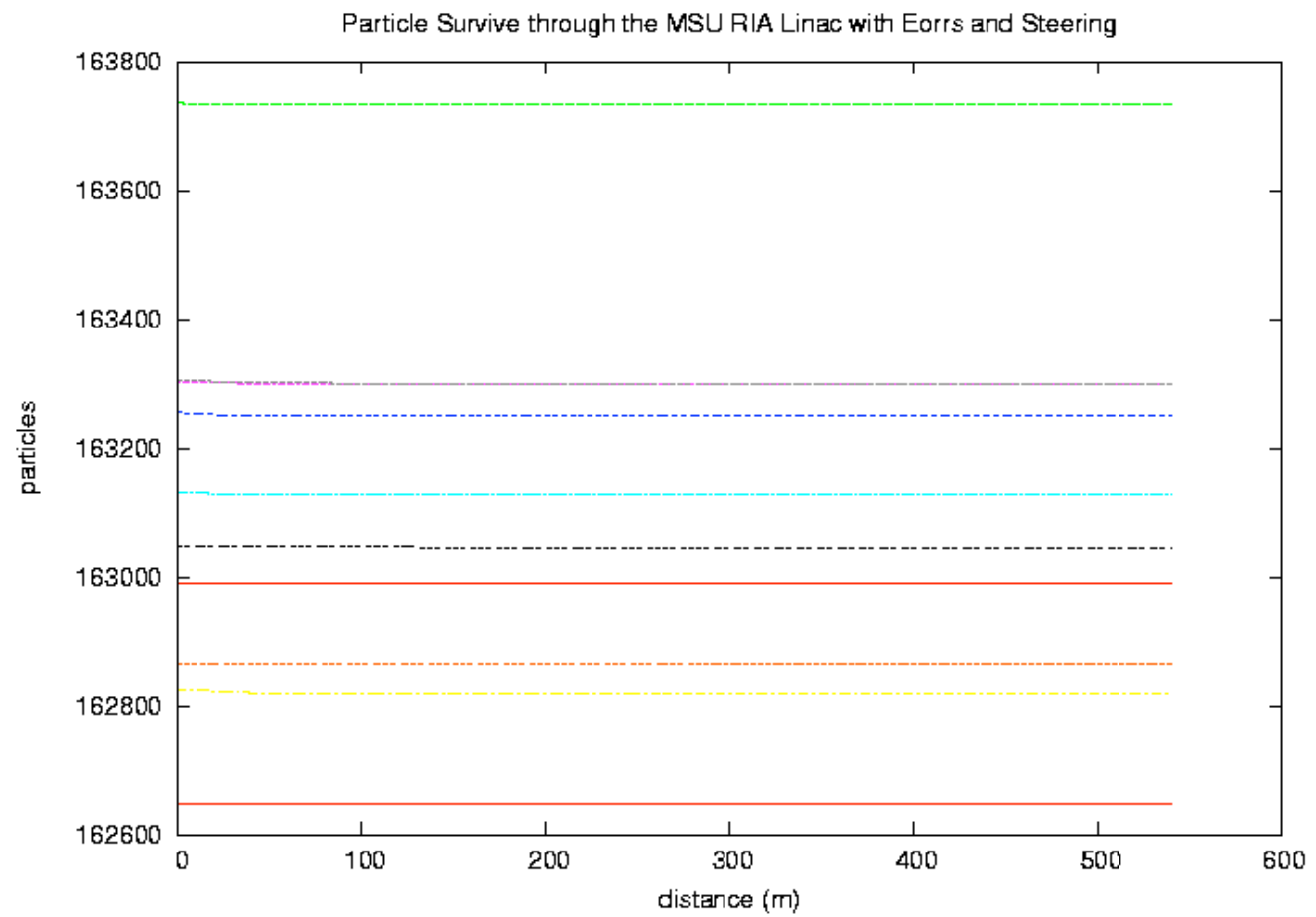

